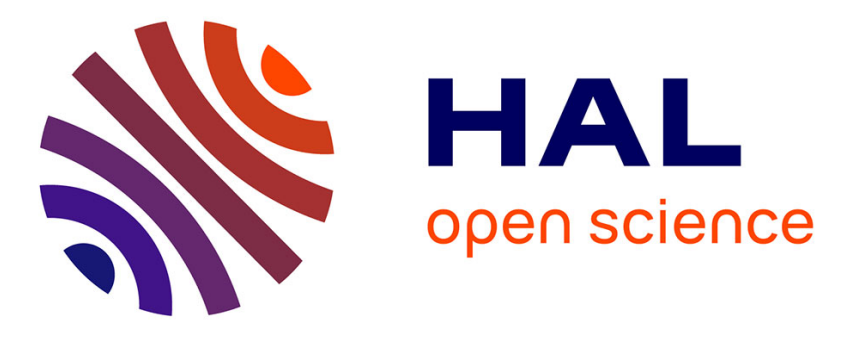

\title{
Control-Induced Time-Scale Separation for Multiterminal High-Voltage Direct Current Systems Using Droop Control
}

Yijing Chen, Miguel Jiménez Carrizosa, Gilney Damm, Françoise

Lamnabhi-Lagarrigue, Ming Li, Yan Li

\section{To cite this version:}

Yijing Chen, Miguel Jiménez Carrizosa, Gilney Damm, Françoise Lamnabhi-Lagarrigue, Ming Li, et al.. Control-Induced Time-Scale Separation for Multiterminal High-Voltage Direct Current Systems Using Droop Control. IEEE Transactions on Control Systems Technology, 2020, 28 (3), pp.967-983. 10.1109/TCST.2019.2901343 . hal-02090569

\section{HAL Id: hal-02090569 https://hal.science/hal-02090569}

Submitted on 4 Apr 2019

HAL is a multi-disciplinary open access archive for the deposit and dissemination of scientific research documents, whether they are published or not. The documents may come from teaching and research institutions in France or abroad, or from public or private research centers.
L'archive ouverte pluridisciplinaire HAL, est destinée au dépôt et à la diffusion de documents scientifiques de niveau recherche, publiés ou non, émanant des établissements d'enseignement et de recherche français ou étrangers, des laboratoires publics ou privés. 


\title{
Control Induced Time-Scale Separation for Multi-Terminal High Voltage Direct Current Systems Using Droop Control
}

\author{
Yijing Chen, Miguel Jiménez Carrizosa, Gilney Damm, Françoise Lamnabhi-Lagarrigue, Ming Li and Yan Li
}

\begin{abstract}
This paper introduces a control induced time-scale separation scheme for a multi-terminal high voltage direct current system, used for large scale integration of renewable energy sources. The main idea is to provide a detailed theoretical analysis, to the long stand practice that consists of empirical design of two control loops for the terminals. Experience has shown that such loops, i.e. current and voltage control loop, when heuristically tuned, often display very different dynamics. In the present paper, singular perturbation theory is applied to give explanation and fundamental analysis on why and how the two control loops work, and how to achieve the timescale separation between various state variables. Mathematical analysis is also carried out to illustrate a clear trade-off between system performance (actuator constraint) and the size of the region of attraction of the controller. Numerical simulations for a system with four terminals are presented to evaluate the system performance and illustrate the theoretical analysis.
\end{abstract}

Index Terms-Droop control, power systems stability, singular perturbation, multi-terminal VSC-HVDC systems.

\section{INTRODUCTION}

W ITH the rapid development of semiconductor devices, multi-terminal high voltage direct current (MTDC) systems based on voltage source converter (VSC) technology have become more and more attractive. Due to their flexibility and controllability, such systems are particularly applicable to the integration of scattered renewable energy sources, such as offshore wind farms, solar plants etc, to the mainland grids [1], [2], [3], [4], [5]. Taking physical considerations into account, the operation range of MTDC systems is limited by the DC voltage which must be kept within a narrow domain of operation [6], [7].

Various DC voltage control strategies are reported in the literature [8], [9], [10], [11], [12], [13], [14]. This paper mainly focus on droop control method [9], [12], [14], [15], [16], [17], [18], which is usually characterized by power vs DC voltage $(P$ vs $U$ ) or current vs DC voltage $(I$ vs $U$ ) curves. In a DC

Y. Chen is with Laboratoire des Signaux et Systèmes on leave from L2S to State Key Laboratory State Key Laboratory of HVDC Electric Power Research Institute, China Southern Power Grid, No. 11, Kexiang Road, Huangpu District, Guangzhou, China e-mail: chenyj@csg.cn.

M. Jiménez Carrizosa is with Universidad Politécnica de Madrid, Spain e-mail: miguel.jimenezcarrizosa@upm.es.

G. Damm is with Laboratoire IBISC, Université d'Evry-Val d'Essonne, France e-mail: gilney.damm@ibisc.fr.

F. Lamnabhi-Lagarrigue is with Laboratoire des Signaux et Systèmes, France e-mail: francoise.lamnabhi-lagarrigue@1ss.supelec.fr.

M. Li and Y. Li are with State Key Laboratory of HVDC Electric Power Research Institute, China Southern Power Grid, No. 11, Kexiang Road, Huangpu District, Guangzhou, China e-mail: liming@csg.cn, liyan@csg.cn. voltage droop control scheme, more than one terminal is used to regulate the DC voltage where the droop gains specify the system operation in steady-state condition and have a great effect on the system transient performance.

Different methodologies of choosing droop gains have been investigated. In [19], a criterion for tuning the droop gains based on the performance specifications is proposed. Reference [15] develops an adaptive droop control scheme according to each terminal's available spare capacity. However, prior studies are usually under the unproven assertion that the dynamics of the inductor currents are much faster than the dynamics of the DC network. Therefore, the currents through the converters are assumed to equal their references and then, the dynamic interaction between $\mathrm{AC}$ and $\mathrm{DC}$ sides of the converters can be neglected. Unfortunately, this assertion, is rarely verified in detail. Acknowledging this fact, the present paper will explore and explain the conditions and limitations of this assertion. To achieve this goal, a full theoretical analysis of the time-scale separation between the system state variables is carried out based on singular perturbation theory [20], which is widely used in the context of the dynamic analysis of power systems [21], [22], [23], [24]. Furthermore, sufficient conditions on the control gains are derived to ensure the locally asymptotic system stability by using Lyapunov theory. Moreover, a trade-off between the system performance and the region of attraction is also presented in the analysis.

The remainder of this paper is outlined as follows. An MTDC system with a generic DC grid topology is modeled in Section II. In Section III, we define the control operation and introduce the control scheme. The main contribution of this paper is presented in Section IV. A detailed theoretical analysis is carried out by means of singular perturbation and Lyapunov theories, from which the system in steady-state condition is discussed and the sufficient conditions on the control gains for stability and time-scale separation are also established. In Section V, numerical simulations for an MTDC system with four terminals are presented to illustrate the theoretical analysis. Conclusions and future work are drawn in Section VI.

Notation: For a matrix $A \in \mathbb{R}^{n \times m}, A_{i j}$ is the element of $A$ in the $i^{\text {th }}$ row and the $j^{\text {th }}$ column. $A(:, i)$ and $A(j,:)$ denote the $i^{\text {th }}$ column and the $j^{\text {th }}$ row of $A$, respectively. A diagonal matrix $A \in \mathbb{R}^{n \times n}$ is represented by $A=\operatorname{diag}\left(a_{i}\right)$, $i=1, \cdots, n . A(l: s, k: h)$ is a submatrix of $A$ where $A(l$ : $s, k: h)_{i j}=A_{(l+i-1)(k+j-1)}$. Given a set of matrix $A_{k} \in$ $\mathbb{R}^{m_{k} \times m_{k}}, k=1, \cdots, n$ and $N=\sum_{k=1}^{n} m_{k}, A=\operatorname{diag}\left(A_{k}\right)$ 


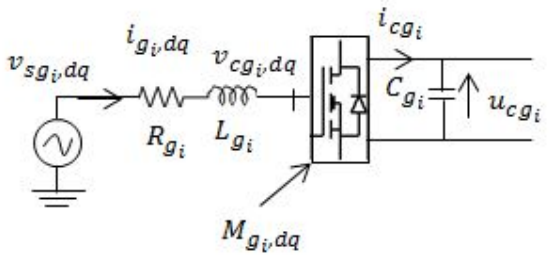

Fig. 1. A simplified configuration of the $i^{\text {th }}$ GVSC terminal.

represents $A \in \mathbb{R}^{N \times N}$ where $A\left(1: m_{1}, 1: m_{1}\right)=A_{1}$ and $A\left(\sum_{j=1}^{k-1} m_{j}+1: \sum_{j=1}^{k} m_{j}, \sum_{j=1}^{k-1} m_{j}+1: \sum_{j=1}^{k} m_{j}\right)=A_{k}$, for $k=2, \cdots, n$, and the other elements of $A$ are zero. The transpose of $\mathrm{A}$ is denoted by $A^{T} \cdot \operatorname{rank}(A)$ means the rank of $A$. $0_{n \times m} \in \mathbb{R}^{n \times m}$ represents the zero matrix, with all its elements equal zero. The zero vector is denoted by $0_{n} \in \mathbb{R}^{n}$. For $x=\left[\begin{array}{lll}x_{1} & \cdots & x_{n}\end{array}\right]^{T} \in \mathbb{R}^{n},\|x\|=\sqrt{x_{1}^{2}+\cdots+x_{n}^{2}}$. For $A \in \mathbb{R}^{n \times n},\|A\|=\sqrt{\lambda_{\max }\left(A^{H} A\right)}$ where $A^{H}$ is the conjugate transpose of $A$ and $\lambda_{\max }(\cdot)$ represents the maximum eigenvalue of matrix $(\cdot) . \mathbb{D}_{x} \subset \mathbb{R}^{n}$ denotes the safe operating domain of variable $x \in \mathbb{R}^{n}$, which is convex. We define the sets $\mathcal{N}=\{1, \cdots, N\}, \mathcal{M}=\{1, \cdots, M\}, \mathcal{L}=\{1, \cdots, L\}$, $\mathcal{P}=\{1, \cdots, P\}, \mathcal{M}_{\mathcal{N}}=\{1+N, \cdots, M+N\}$ and $\mathcal{T}=\{1, \cdots, N+M+P\}$. Unless otherwise noted, $i, j$, $k$ and $h$ denote " $\forall i \in \mathcal{N} ", " \forall j \in \mathcal{M} ", " \forall k \in \mathcal{L}$ " $\forall$ and " $\forall$ $h \in \mathcal{P}$ ", respectively. $\bar{x}$ represents the value of $x$ in steadystate condition and the initial value of $x$ is denoted by $x\left(t_{0}\right)$. The prescribed value of the variable $x$ is denoted as $x^{o}$. Any complex matrix $A$ can be expressed as $A=\mathcal{H}(A)+\mathcal{S}(A)$ where $\mathcal{H}(A)=1 / 2\left(A+A^{H}\right)$ is a hermitian matrix and $\mathcal{S}(A)=1 / 2\left(A-A^{H}\right)$ is an anti-hermitian matrix. $\mathcal{R}(A)_{l}$ is a reduced matrix by deleting the $l^{\text {th }}$ row of matrix $A$.

\section{Modeling OF AN MTDC System}

In this paper, we consider an MTDC system composed of $N$ grid connected VSC (GVSC) terminals, $M$ wind farm connected VSC (WVSC) terminals and a generic DC grid topology. The AC side of all converters connected to the MTDC system is modeled in a synchronously rotating reference $d q$ frame. By convention, the active power on the $\mathrm{AC}$ side and the current through the phase reactor are positive if they flow from the AC side to the DC side via the VSC.

\section{A. GVSC terminal modeling}

A similar method as in [25] for modeling the GVSC terminal in a $d q$ reference frame is briefly described in this section. The configuration of the $i^{\text {th }}$ GVSC is shown in Fig. 1 where the currents $i_{g_{i}, d q}$ flow through the phase reactor made up of an aggregated resistance $R_{g_{i}}$ and an aggregated inductance $L_{g_{i}}$. The AC network is modeled by an ideal threephase AC source represented by $v_{s g_{i}, d q}$ at the frequency $f_{g_{i}}$. According to Kirchhoff's circuit laws, the dynamics of $i_{g_{i}, d q}$ can be expressed by:

$$
\begin{aligned}
& \frac{d i_{g_{i} d}}{d t}=-\frac{R_{g_{i}}}{L_{g_{i}}} i_{g_{i} d}+\omega_{g_{i}} i_{g_{i} q}+\frac{v_{s g_{i} d}}{L_{g_{i}}}-\frac{u_{c g_{i}}}{2 L_{g_{i}}} M_{g_{i} d} \\
& \frac{d i_{g_{i} q}}{d t}=-\frac{R_{g_{i}}}{L_{g_{i}}} i_{l q}-\omega_{g_{i}} i_{g_{i} d}+\frac{v_{s g_{i} q}}{L_{g_{i}}}-\frac{u_{c g_{i}}}{2 L_{g_{i}}} M_{g_{i} q}
\end{aligned}
$$

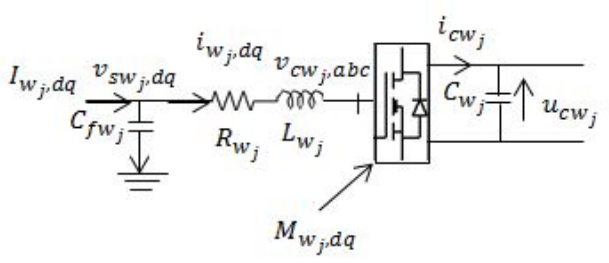

Fig. 2. A simplified configuration of the $j^{\text {th }}$ WVSC terminal.

where $M_{g_{i}, d q}$ are the modulation indices for the converter control [26] and $\omega_{g_{i}}=2 \pi f_{g_{i}}$. For the sake of simplicity, the rotating angle of Park's transformation is usually chosen such that the $d$-axis is aligned to the phase $a$ of AC voltage, which results in $v_{s g_{i} d}=V_{g_{i} \text {.rms }}$ and $v_{s g_{i} q}=0$ where $V_{g_{i} \text {.rms }}$ is the root mean square value of $\mathrm{AC}$ network voltage. In addition, the active and reactive power flows at the

$$
P_{g_{i}}=\frac{3}{2} v_{s g_{i} d} i_{g_{i} d}, Q_{g_{i}}=-\frac{3}{2} v_{s g_{i} d} i_{g_{i} q}
$$

\section{B. WVSC terminal modeling}

Figure 2 depicts a simplified configuration of the $j^{\text {th }}$ WVSC terminal where the wind farm is modeled as a controlled current source represented by $I_{w_{j}, d q}$ [25], [27]. A simple capacitor $C_{f w_{j}}$ operates as a high frequency $\mathrm{AC}$ filter, used to eliminate unacceptable harmonics.

Similar to the modeling of GVSC, the dynamics of the $d q$ current $i_{w_{j}, d q}$ flowing into the phase reactor represented by a resistance $R_{w_{j}}$ and an inductance $L_{w_{j}}$ are given by:

$$
\begin{aligned}
& \frac{d i_{w_{j} d}}{d t}=-\frac{R_{w_{j}}}{L_{w_{j}}} i_{w_{j} d}+\omega_{w_{j}} i_{w_{j} q}+\frac{v_{s w_{j} d}}{L_{w_{j}}}-\frac{u_{c w_{j}}}{2 L_{w_{j}}} M_{w_{j} d} \\
& \frac{d i_{w_{j} q}}{d t}=-\frac{R_{w_{j}}}{L_{w_{j}}} i_{w_{j} q}-\omega_{w_{j}} i_{w_{j} d}+\frac{v_{s w_{j} q}}{L_{w_{j}}}-\frac{u_{c w_{j}}}{2 L_{w_{j}}} M_{w_{j} q}
\end{aligned}
$$

The AC voltages at the PCC, $v_{s w_{j}, d q}$, are modeled by:

$$
\begin{aligned}
& \frac{d v_{s w_{j} d}}{d t}=\omega_{w_{j}} v_{s w_{j} q}+\frac{1}{C_{f w_{j}}}\left(I_{w_{j} d}-i_{w_{j} d}\right) \\
& \frac{d v_{s w_{j} q}}{d t}=-\omega_{w_{j}} v_{s w_{j} d}+\frac{1}{C_{f w_{j}}}\left(I_{w_{j} q}-i_{w_{j} q}\right)
\end{aligned}
$$

with $\omega_{w_{j}}=2 \pi f_{w_{j}}$. In addition, the frequency $f_{w_{j}}$ can be freely set since there is no need to synchronize the rotor frequency with the general grid frequency.

The active and the reactive power at the PCC are expressed as:

$$
\begin{aligned}
& P_{w_{j}}=\frac{3}{2}\left(v_{s w_{j} d} i_{w_{j} d}+v_{s w_{j} q} i_{w_{j} q}\right) \\
& Q_{w_{j}}=\frac{3}{2}\left(v_{s w_{j} q} i_{w_{j} d}-v_{s w_{j} d} i_{w_{j} q}\right)
\end{aligned}
$$

Remark 1: Taking physical considerations into account, all the modulation indices $M_{d q}$ are limited by $M_{d}^{2}+M_{q}^{2} \leq 1$. In addition, the active power of the wind farm $P_{w_{j}}$ are always non-negative, i.e. $P_{w_{j}} \geq 0$.

\section{DC grid modeling}

We consider a generic DC network topology formed by $N$ GVSC nodes, $M$ WVSC nodes, $P$ intermediate nodes and $L$ transmission branches [19]. Each node is characterized by its corresponding DC voltage $\left(u_{c g_{i}}, u_{c w_{j}}, u_{c t_{h}}\right)$ and DC capacitor $\left(C_{g_{i}}, C_{w_{j}}, C_{t_{h}}\right)$. The transmission line $l_{k}$ is modeled 


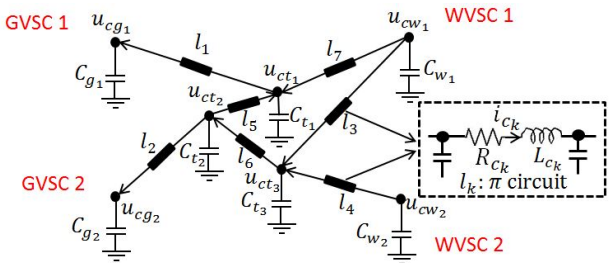

Fig. 3. An example of the DC grid.

by a lumped $\pi$-equivalent circuit consisting of an aggregated resistance and an aggregated inductance $\left(R_{c_{k}}+j L_{c_{k}}\right)$. The branch current of $l_{k}$ is denoted as $i_{c_{k}}$. Every branch is used to connect two adjacent nodes and every node can be connected to a number of transmission lines. An example of such DC grid with two GVSC nodes $\left(u_{c g_{1,2}}\right)$, two WVSC nodes $\left(u_{c w_{1,2}}\right)$, three intermediate nodes $\left(u_{c t_{1,2,3}}\right)$ and seven branches $\left(i_{c_{k}}\right.$, $k=1, \cdots, 7)$ is depicted in Fig. 3 .

To better understand the properties of the DC network, we analyze its topology with the help of graph theory. In this paper, we study a class of DC networks which can be represented by a weakly connected directed graph $G$ without self-loops. This graph is labeled by $G=(V, E) . V=\left\{V_{1}, V_{2}, V_{3}\right\}$ is the set of the vertices where $V_{1}=\left\{v_{1}, \cdots, v_{N}\right\}, V_{2}=$ $\left\{v_{N+1}, \cdots, v_{N+M}\right\}$ and $V_{3}=\left\{v_{N+M+1}, \cdots, v_{N+M+P}\right\}$ correspond to the $N$ GVSC nodes, the $M$ WVSC nodes and the $P$ intermediate nodes, respectively. $E=\left\{e_{1}, \cdots, e_{L}\right\}$ is the set of the edges mapped to the $L$ circuit branches. The incidence matrix of $G=(V, E)$ is denoted by $H \in \mathbb{R}^{(N+M+P) \times L}$ and its element in the $l^{\text {th }}$ row and the $k^{\text {th }}$ column, i.e. $H_{l k}$, satisfies

$$
H_{l k}=\left\{\begin{aligned}
1 \quad & \text { if the branch current of } e_{k} \text { flows } \\
& \text { into the node } v_{l}, \\
-1 \quad & \text { if the branch current of } e_{k} \text { flows } \\
\text { from the node } v_{l}, & \text { otherwise. }
\end{aligned}\right.
$$

In general, the incidence matrix $H$ of the weakly connected directed graph $G$ without self-loops has the following features:

- Since the directed graph $G$ is weakly connected, the numbers of the vertices and the edges must satisfy $L \geq(N+M+P)-1$.

- Every edge (transmission line) can only connect two vertices (nodes) and hence, each column of $H$ has only two non-zero elements, 1 and -1 .

Based on the above characteristics, we have the following results whose proofs are referred in [28], [29].

Lemma 1: The vectors $H(1,:), H(2,:), \ldots, H(N+M+P,:)$ are linearly dependent and the rank of $H$ is $N+M+P-1$. In addition, $\sum_{i=1}^{N+M+P} H(i,:)=0_{L}^{T}$.

Lemma 2: If any one row is removed from $H$, for example, $H(l,:), l \in \mathcal{T}$, we obtain a reduced incidence matrix $\mathcal{R}(H)_{l}$ whose rank is still $N+M+P-1$. It means that the remaining $(N+M+P-1)$ row vectors are linearly independent.
Considering the lumped $\pi$-circuit model [30], the dynamics of the DC grid can be written in matrix expression form:

$$
\dot{z}=A z+\vartheta
$$

where $z, \vartheta \in \mathbb{R}^{N+M+P+L}$ are given by:

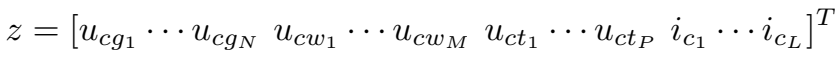

$$
\begin{aligned}
& \vartheta=\left[\frac{i_{c g_{1}}}{C_{g_{1}}} \cdots \frac{i_{c g_{N}}}{C_{g_{N}}} \frac{i_{c w_{1}}}{C_{w_{1}}} \cdots \frac{i_{c w_{M}}}{C_{w_{M}}} 0_{(P+L)}^{T}\right]^{T}
\end{aligned}
$$

The matrix $A$ is of the form:

$$
A=\left[\begin{array}{cc}
0_{(N+M+P) \times(N+M+P)} & C^{-1} H \\
-L^{-1} H^{T} & -L^{-1} R
\end{array}\right]
$$

where $C \in \mathbb{R}^{(N+M+P) \times(N+M+P)}$ and $L, R \in \mathbb{R}^{L \times L}$ are the capacitor, inductance and resistance matrices respectively, which are given by:

$$
\begin{aligned}
& C=\operatorname{diag}\left(C_{g_{1}} \cdots C_{g_{N}} C_{w_{1}} \cdots C_{w_{M}} C_{p_{1}} \cdots C_{p_{P}}\right) \\
& L=\operatorname{diag}\left(L_{c_{1}} \cdots L_{c_{L}}\right), \quad R=\operatorname{diag}\left(R_{c_{1}} \cdots R_{c_{L}}\right)
\end{aligned}
$$

Due to the power balance on both sides of the converters, $i_{c g_{i}}$ and $i_{c w_{j}}$ can be deduced as:

$$
\begin{aligned}
i_{c g_{i}}=\frac{P_{g, i}}{u_{c g_{i}}}=\frac{3\left(v_{s g_{i} d} i_{g_{i} d}+v_{s g_{i} q} i_{g_{i} q}\right)}{2 u_{c g_{i}}}=\frac{3 v_{s g_{i} d} i_{g_{i} d}}{2 u_{c g_{i}}} \\
i_{c w_{j}}=\frac{P_{w, j}}{u_{c w_{j}}}=\frac{3\left(v_{s w_{j} d} i_{w_{j} d}+v_{s w_{j} q} i_{w_{j} q}\right)}{2 u_{c w_{j}}}
\end{aligned}
$$

while the losses of the phase reactor and the semiconductors are neglected [31], [32].

Remark 2: In [26], it is indicated that the operation of the converter is only valid in a limited region. If the operation is beyond the safe operating region, it will damage the device (for example, the DC voltage exceeds a maximum value). Therefore, we restrict the next theoretical analysis to a finite safe operating region where $i_{g_{i}, d q} \in \mathbb{D}_{i_{g_{i}, d q}}, i_{w_{j}, d q} \in \mathbb{D}_{i_{w_{j}, d q}}$, $u_{c g_{i}} \in \mathbb{D}_{u_{c g_{i}}}, u_{c w_{j}} \in \mathbb{D}_{u_{c w_{j}}}, u_{c t_{h}} \in \mathbb{D}_{u_{c t_{h}}}$ and $i_{c_{k}} \in \mathbb{D}_{i_{c_{k}}}$.

\section{VSC OPERATION AND CONTROL STRUCTURE}

\section{A. VSC operation}

To ensure the normal operation of windfarm connections via the DC grid, all of the WVSCs are required to keep their AC voltage magnitudes and frequencies constant at the PCCs. This can be achieved by regulating $v_{s w_{j}, d q}$ at their setpoints $v_{s w_{j}, d q}^{o}$ [27]. Since the semiconductor devices in VSC are very sensitive to overvoltage, it is very important to restrict the DC voltage to an acceptable band. To control the DC voltage, the present work uses a droop strategy [33], which is widely applied in the context of control of MTDC systems. This control method has the advantage of sharing the duty of eliminating the power imbalance of the DC grid between several terminals. Additionally, it takes actions only based on local information without remote communication. In our case, we consider that every GVSC terminal is equipped with the DC droop controller. Moreover, the reactive powers of all the GVSCs at the PCCs, i.e. $Q_{g_{i}}$, are required to track the reference values $Q_{g_{i}}^{o}$. 


\section{B. Control structure}

The proposed control structure is inspried on the so called vector control method [27], [34]. This control strategy widely used in industry is commonly applied to the VSCs and has the advantage of a simple design procedure. The control design consists of two loops, i.e. a fast and a slow control loop. The reference sent to the fast control loop is derived from the slow control loop. It is assumed that the dynamics of state variables in the fast control loop are much faster than the dynamics of the state variables in the slow control loop. Because of this dynamic separation in different time scales, the two loops can be designed separately. We have a similar approach as in [19], [27] for building the control structure.

1) Design of the fast control loop: As described in (1) and (3), the control inputs $M_{(\cdot), d q}$ are directly collocated with the $d q$ currents $i_{(\cdot), d q}$. Hence, a sub-controller can be developed such that $i_{(\cdot), d q}$ quickly converge to their reference trajectories $i_{(\cdot), d q}^{*}$ yet to be designed.

We first define the $d q$ current tracking errors by:

$$
\begin{gathered}
e_{i_{g_{i}, d q}}=i_{g_{i}, d q}-i_{g_{i}, d q}^{*} \\
e_{i_{w_{j}, d q}}=i_{w_{j}, d q}-i_{w_{j}, d q}^{*}
\end{gathered}
$$

With the introduction of the integral tracking errors $e_{I i_{g_{i}, d q}}$ and $e_{I i_{w_{j}, d q}}$, an augmented $d q$ current subsystem is then generated by:

$$
\begin{aligned}
\frac{d e_{I i_{g_{i}} d}}{d t} & =e_{i_{g_{i} d}} \\
\frac{d e_{i_{i} d}}{d t} & =-\frac{R_{g_{i}}}{L_{g_{i}}} i_{g_{i} d}+\omega_{g_{i}} i_{g_{i} q}+\frac{v_{s g_{i} d}}{L_{g_{i}}} \\
& -\frac{M_{g_{i} d} u_{c g_{i}}}{2 L_{g_{i}}}-\frac{d i_{g_{i} d}^{*}}{d t} \\
\frac{d e_{I i_{i} q}}{d t} & =e_{i_{g_{i} q}} \\
\frac{d e_{i_{i} q} q}{d t} & =-\frac{R_{g_{i}}}{L_{g_{i}}} i_{g_{i} q}-\omega_{g_{i}} i_{g_{i} d}+\frac{v_{s g_{i} q}}{L_{g_{i}}} \\
& -\frac{M_{g_{i} q} u_{c g_{i}}}{2 L_{g_{i}}}-\frac{d i_{g_{i} q}^{*}}{d t} \\
\frac{d e_{I i_{w_{j}} d}}{d t} & =e_{i_{w_{j} d}} \\
\frac{d e_{i_{w_{j}} d}}{d t} & =-\frac{R_{w_{j}}}{L_{w_{j}}} i_{w_{j} d}+\omega_{w_{j}} i_{w_{j} q}+\frac{v_{s w_{j} d}}{L_{w_{j}}} \\
& -\frac{m_{w_{j} d} u_{c w_{j}}}{2 L_{w_{j}}}-\frac{d i_{w_{j} d}^{*}}{d t} \\
\frac{d e_{I i_{w_{j} q} q}}{d t} & =e_{i_{w_{j} q}} \\
\frac{d e_{i_{w_{j}} q}}{d t} & =-\frac{R_{w_{j}}}{L_{w_{j}}} i_{w_{j} q}-\omega_{w_{j}} i_{w_{j} d}+\frac{v_{s w_{j} q}}{L_{w_{j}}} \\
& -\frac{M_{w_{j} q} u_{c w_{j}}}{2 L_{w_{j}}}-\frac{d i_{w_{j} q}^{*}}{d t}
\end{aligned}
$$

In practice, the dynamics of $i_{g_{i}, d q}^{*}$ and $i_{w_{j}, d q}^{*}$ are usually neglected and considered as slow compared to $i_{g_{i}, d q}$ and $i_{w_{j}, d q}$. Thus, the modulation indices can then be designed as:

$$
\begin{aligned}
M_{g_{i} d}= & \frac{2 L_{g_{i}}}{u_{c g_{i}}}\left(-\frac{R_{g_{i}}}{L_{g_{i}}} i_{g_{i} d}+\omega_{g_{i}} i_{g_{i} q}+\frac{v_{s g_{i} d}}{L_{g_{i}}}\right. \\
& \left.+k_{1 g_{i} d} e_{i_{g_{i} d}}+k_{2 g_{i} d} e_{I i_{g_{i} d}}\right) \\
M_{g_{i} q}= & \frac{2 L_{g_{i}}}{u_{c g_{i}}}\left(-\frac{R_{g_{i}}}{L_{g_{i}}} i_{g_{i} q}-\omega_{g_{i}} i_{g_{i} d}+\frac{v_{s g_{i} q}}{L_{g_{i}}}\right. \\
& \left.+k_{1 g_{i} q} e_{i_{g_{i} q}}+k_{2 g_{i} q} e_{I i_{g_{i} q}}\right) \\
M_{w_{j} d}= & \frac{2 L_{w_{j}}}{u_{c w_{j}}}\left(-\frac{R_{w_{j}}}{L_{w_{j}}} i_{w_{j} d}+\omega_{w_{j}} i_{w_{j} q}+\frac{v_{s w_{j} d}}{L_{w_{j}}}\right. \\
& \left.+k_{1 w_{j} d} e_{i_{w_{j} d}}+k_{2 w_{j} d} e_{I i_{w_{j} d}}\right) \\
M_{w_{j} q}= & \frac{2 L_{w_{j}}}{u_{c w_{j}}}\left(-\frac{R_{w_{j}}}{L_{w_{j}}} i_{w_{j} q}-\omega_{w_{j}} i_{w_{j} d}+\frac{v_{s w_{j} q}}{L_{w_{j}}}\right. \\
& \left.+k_{1 w_{j} q} e_{i_{w_{j} q}}+k_{2 w_{j} q} e_{I i_{w_{j} q}}\right)
\end{aligned}
$$

with positive control gains $k_{1,2 g_{i}, d q}$ and $k_{1,2 w_{j}, d q}$.

Remark 3: It is clear that the equilibrium (the origin) of the augmented $d q$ current subsystem (11) is exponentially stable under the sub-controller (12) when $i_{g_{i}, d q}^{*}$ and $i_{w_{j}, d q}^{*}$ are constant or slowly varying.

2) Design of slow control loop: The main task of the slow control loop is to provide the slowly varying $d q$ current references to the fast control loop. Most importantly, the $d q$ current references need to be designed such that all control objectives are achieved. Since the operations of GVSCs and WVSCs are different, the design principles of $i_{g_{i}, d q}^{*}$ and $i_{w_{j}, d q}^{*}$ are also different. Assuming that $i_{w_{j}, d q}$ quickly converge to their reference trajectories $i_{w_{j} d q}^{*}$ under the sub-controller (12), the AC voltage dynamics (4) of the wind farm at the PCC become:

$$
\begin{aligned}
& \frac{d v_{s w_{j} d}}{d t}=\omega_{w_{j}} v_{s w_{j} q}+\frac{1}{C_{f w_{j}}}\left(I_{w_{j} d}-i_{w_{j} d}^{*}\right) \\
& \frac{d v_{s w_{j} q}}{d t}=-\omega_{w_{j}} v_{s w_{j} d}+\frac{1}{C_{f w_{j}}}\left(I_{w_{j} q}-i_{w_{j} q}^{*}\right)
\end{aligned}
$$

where $i_{w_{j} d q}^{*}$ can be viewed as the control inputs. We call (13) the reduced model of (4) since the dynamics of $i_{w_{j}, d q}$ are neglected and $i_{w_{j}, d q}$ are replaced by $i_{w_{j}, d q}^{*}$. The control objective of WVSCs is to keep $v_{s w_{j}, d q}$ at their reference values $v_{s w_{j}, d q}^{o}$ in spite of variations in the power productions of the wind farms. We then want to develop $i_{w_{j}, d q}^{*}$ in such a way that $v_{s w_{j}, d q}$ in (13) can be controlled at $v_{s w_{j}, d q}^{o}$. Similar to the design procedure of the fast control loop, we define the output tracking errors of voltage at the PCC as:

$$
e_{v_{s w_{j}, d q}}=v_{s w_{j}, d q}-v_{s w_{j}, d q}^{o}
$$

We then augment (13) by taking the integral tracking errors into account:

$$
\begin{aligned}
& \frac{d e_{I v_{s w_{j} d}}}{d t}=e_{v_{s w_{j} d}} \\
& \frac{d e_{v_{s w_{j} d}}}{d t}=\frac{1}{C_{f w_{j}}}\left(I_{w_{j} d}+C_{f w_{j}} \omega_{w_{j}} v_{s w_{j} q}-i_{w_{j} d}^{*}\right)-\frac{d v_{s w_{j} d}^{o}}{d t} \\
& \frac{d e_{I v_{s w_{j} q}}}{d t}=e_{v_{s w_{j} q}} \\
& \frac{d e_{v_{s w_{j} d q}}}{d t}=\frac{1}{C_{f w_{j}}}\left(I_{w_{j} q}-C_{f w_{j}} \omega_{w_{j}} v_{s w_{j} d}-i_{w_{j} q}^{*}\right)-\frac{d v_{s w_{j} q}^{o}}{d t}
\end{aligned}
$$

Since the prescribed values $v_{s w_{j}, d q}^{o}$ are constant, we have $\frac{d v_{s w_{j}, d q}^{o}}{d t}=0$. Finally, $i_{w_{j}, d q}^{*}$ can be designed as:

$$
\begin{aligned}
& i_{w_{j} d}^{*}=I_{w_{j} d}+C_{f w_{j}}\left(k_{1 d_{j}} e_{v_{s w_{j} d}}+k_{2 d_{j}} e_{I v_{s w_{j} d}}+\omega_{w_{j}} v_{s w_{j} q}\right) \\
& i_{w_{j} q}^{*}=I_{w_{j} q}+C_{f w_{j}}\left(k_{1 q_{j}} e_{v_{s w_{j} q}}+k_{2 q_{j}} e_{I v_{s w_{j} q}}-\omega_{w_{j}} v_{s w_{j} d}\right)
\end{aligned}
$$

with positive $k_{1, d q_{j}}$ and $k_{2, d q_{j}}$ such that the equilibrium of the augmented model (15) is exponentially stable.

As mentioned in the foregoing part, in order to protect the components of the VSCs, the DC voltage must be maintained within a narrow safe operating region during the power transmission process. This can be achieved in the case that each GVSC terminal is equipped with a droop controller. Therefore, all GVSC terminals participate in the regulation of the DC voltage in case of power imbalance. The droop control is usually expressed by DC voltage versus active power characteristic [33]:

$$
P_{g_{i}}=P_{g_{i}}^{o}+K_{d_{i}}\left(u_{c g_{i}}^{o}-u_{c g_{i}}\right)
$$


where $u_{c g_{i}}^{o}$ and $P_{g_{i}}^{o}$ refer to the reference values of DC voltage and active power respectively. The droop gain $K_{d_{i}}$ is the ratio of the change in DC voltage to the change in active power. According to (17), the varying reference for $i_{g_{i} d}$ can be expressed by:

$$
i_{g_{i} d}^{*}=i_{g_{i} d}^{o}-k_{d_{i}}\left(u_{c g_{i}}-u_{c g_{i}}^{o}\right)
$$

where $i_{g_{i} d}^{o}$ is obtained from (2) and expressed as:

$$
i_{g_{i} d}^{o}=\frac{2 P_{g_{i}}^{o}}{3 v_{s g_{i} d}}
$$

In addition, the relation between $K_{d_{i}}$ and $k_{d_{i}}$ is given by:

$$
k_{d_{i}}=\frac{2}{3} \frac{1}{v_{s g_{i} d}} K_{d_{i}}
$$

Considering $Q_{g_{i}}$ in (2), the reactive power can be directly regulated by $i_{g_{i} q}$. Hence, $i_{g_{i} q}^{*}$ can be designed as:

$$
i_{g_{i} q}^{*}=-\frac{2}{3} \frac{Q_{g_{i}}^{o}}{v_{s g_{i} d}} \triangleq i_{g_{i} q}^{o}
$$

Remark 4: All of the setpoints, i.e. $u_{c g_{i}}^{o}, P_{g_{i}}^{o} Q_{g_{i}}^{o}$ and $v_{s w_{j}, d q}^{o}$, are provided by a higher level controller. Their choice should consider several factors [33], [35]. It is still an open issue to set the proper values for these setpoints so that the power flow of the MTDC system in steady-state condition corresponds to the predetermined one from the power flow dispatch. This problem is out of scope of this paper but can be referred in [7]. In this paper, these setpoints are considered as known constants and hence, $\frac{d v_{s w_{j}, d q}^{o}}{d t}$ in (15) are equal to zero.

Remark 5: In Remark 3, when $i_{(\cdot), d q}^{*}$ are slowly varying, $i_{(\cdot), d q}$ can quickly converge to the quasi-steady states $i_{(\cdot), d q}^{*}$ As seen in (16), (18) and (19), in order to obtain slowly varying $i_{(\cdot), d q}^{*}$, the dynamics of $v_{s w_{j}, d q}$ and $z$ should be slow enough comparing to $i_{(\cdot), d q}$. It is obvious that the dynamics of $v_{s w_{j}, d q}$ and $z$ strongly relay on $k_{1, d q_{j}}, k_{2, d q_{j}}$ and $k_{d_{i}}$. We infer that $v_{s w_{j}, d q}$ and $z$ could be slowly varying by properly tuning $k_{1, d q_{j}}, k_{2, d q_{j}}$ and $k_{d_{i}}$. This will be verified in Section IV.

\section{THEORETICAL ANALYSIS}

The control design concept presented in the previous section is mainly based on the assumption that a dynamic separation in time scales is possible to be imposed between the fast and slow control loops. As a consequence, $z$ and $v_{s w_{j}, d q}$ can be considered as constant in the fast control loop, while the $d q$ currents can be replaced by their references in the slow control loop.

In this section, we give a detailed theoretical analysis to demonstrate the validity of the time-scale separation assumption by means of singular perturbation theory. Furthermore, sufficient conditions on the control gains for ensuring the system stability are also derived based on Lyapunov theory.

\section{A. Equilibrium Analysis}

Before going further, we give a brief analysis of the equilibruim point. Substituting the controller composed by (12), (16) , (18) and (19) into the plant model described by (1), (3), (4) and (7) leads to the following augmented closed-loop system:

$$
\begin{aligned}
\frac{d e_{I i_{g_{i} d}}}{d t} & =e_{i_{g_{i} d}} \\
\frac{d i_{g_{i} d}}{d t} & =-k_{1 g_{i} d} e_{i_{g_{i} d}}-k_{2 g_{i} d} e_{I i_{g_{i} d}} \\
\frac{d e_{I i_{g_{i}} q}}{d t} & =e_{i_{g_{i} q}} \\
\frac{d i_{g_{i} q}}{d t} & =-k_{1 g_{i} q} e_{i_{g_{i} q}}-k_{2 g_{i} q} e_{I i_{g_{i} q}} \\
\frac{d e_{I i_{w_{j}}}}{d t} & =e_{i_{w_{j} d}} \\
\frac{d i_{w_{j} d}}{d t} & =-k_{1 w_{j} d} e_{i_{w_{j} d}}-k_{2 w_{j} d} e_{I i_{w_{j} d}} \\
\frac{d e_{I w_{w_{j}} q}}{d t} & =e_{i_{w_{j} q}} \\
\frac{d i_{w_{j} q}}{d t} & =-k_{1 w_{j} q} e_{i_{w_{j} q}}-k_{2 w_{j} q} e_{I i_{w_{j} q} q} \\
\frac{d e_{I v_{s w_{j} d}}}{d t} & =e_{v_{s w_{j} d}} \\
\frac{d v_{s w_{j} d}}{d t} & =-k_{1 d_{j}} e_{v_{s w_{j} d}}-k_{2 d_{j}} e_{I v_{s w_{j} d}}-\frac{e_{i_{w_{j} d} d}}{C_{f w_{j}}} \\
\frac{d e_{I v_{s w_{j} q}}}{d t} & =e_{v_{s w_{j} q}} \\
\frac{d v_{s w_{j} q}}{d t} & =-k_{1 q_{j}} e_{v_{s w_{j} q}}-k_{2 q_{j}} e_{I v_{s w_{j} q}}-\frac{e_{i w_{j} q}}{C_{f w_{j}}} \\
\dot{z} & =A z+\vartheta
\end{aligned}
$$

It will be more convenient in the following analysis to divide $\vartheta$ into two parts as:

$$
\vartheta=\vartheta_{g}+\vartheta_{w}
$$

Recalling $i_{c g_{i}}$ in (9), $i_{c w_{j}}$ in (10), $i_{w_{j}, d q}^{*}$ in (16) and $i_{g_{i}, d q}^{*}$ in (18), (19), the new variables $\vartheta_{g}$ and $\vartheta_{w}$ are given by:

$$
\begin{aligned}
& \vartheta_{g}=\vartheta_{g_{1}}+\vartheta_{g_{2}}+\vartheta_{g_{3}} \\
& \vartheta_{w}=\vartheta_{w_{1}}+\vartheta_{w_{2}}+\vartheta_{w_{3}}+\vartheta_{w_{4}}
\end{aligned}
$$

with

$$
\begin{aligned}
& \vartheta_{g_{1}}(i)= \begin{cases}\frac{3}{2 C_{g_{i}}} \frac{v_{s g_{i} d} i_{g_{i} d}^{o}+v_{s g_{i} q} i_{g_{i} q}^{o}}{u_{c g_{i}}}, & i \in \mathcal{N} \\
0 . & \text { otherwise }\end{cases} \\
& \vartheta_{g_{2}}(i)= \begin{cases}-\frac{3}{2 C_{g_{i}}} v_{s g_{i} d} k_{d_{i}} \frac{u_{c g_{i}}-u_{c g_{i}}^{o}}{u_{c g_{i}}}, & i \in \mathcal{N} \\
0 . & \text { otherwise }\end{cases} \\
& \vartheta_{g_{3}}(i)= \begin{cases}\frac{3}{2 C_{g_{i}}} \frac{v_{s g_{i} d} e_{i_{g_{i} d}}+v_{s g_{i} q} e_{i_{g_{i} q}}}{u_{c g_{i}}}, & i \in \mathcal{N} \\
0 . & \text { otherwise }\end{cases}
\end{aligned}
$$

and

$$
\begin{aligned}
& \vartheta_{w_{1}}(j+N)= \begin{cases}\frac{3}{2 C_{w_{j}}} \frac{v_{s w_{j} d}^{o} I_{w_{j} d}+v_{s w_{j} q}^{o} I_{w_{j} q}}{u_{c w_{j}}}, & j \in \mathcal{M} \\
0 . & \text { otherwise }\end{cases} \\
& \vartheta_{w_{2}}(j+N)=\left\{\begin{array}{c}
\frac{3}{2 C_{w_{j}}}\left[\frac{v_{s w_{j} d}^{o} C_{f w_{j}} \omega_{w_{j}} e_{v_{s w_{j} q}}+e_{v_{s w_{j} d}} I_{w_{j}} d}{u_{c w_{j}}}\right. \\
+\frac{e_{v_{s w_{j} q}} I_{w_{j} q}-v_{s w_{j} q}^{o} C_{f w_{j}} \omega_{w_{j}} e_{v_{s w_{j} d}}}{u_{c w_{j}}} \\
j \in \mathcal{M} \\
0 . \\
\text { otherwise }
\end{array}\right.
\end{aligned}
$$




$$
\begin{aligned}
& \vartheta_{w_{3}}(j+N)=\left\{\begin{array}{c}
\frac{3}{2 C_{w_{j}}}\left[\frac{v_{s w_{j} d}\left(k_{1 d_{j}} e_{v_{s w_{j} d}}+k_{2 d_{j}} e_{I v_{s w_{j} d}}\right)}{u_{c w_{j}}}\right. \\
\left.+\frac{v_{s w_{j} q}\left(k_{1 q_{j}} e_{v_{s w_{j} q}}+k_{2 q_{j}} e_{I v_{s w_{j} q}}\right)}{u_{c w_{j}}}\right], \\
j \in \mathcal{M}
\end{array}\right. \\
& 0 . \\
& \vartheta_{w_{4}}(j+N)=\left\{\begin{array}{l}
\frac{3}{2 C_{w_{j}}} \frac{v_{s w_{j} d} e_{i_{w_{j} d}}+v_{s w_{j} q} e_{i_{w_{j} q}}}{u_{c w_{j}}}, j \in \mathcal{M} \\
0 . \quad \text { otherwise }
\end{array}\right.
\end{aligned}
$$

Remark 6: It is obvious that, if the $d q$ currents quickly enter their respective manifolds, i.e. $e_{i_{g_{i}, d q}} \rightarrow 0$ and $e_{i_{w_{j}, d q}} \rightarrow 0$, $\vartheta_{g_{3}}$ and $\vartheta_{w_{4}}$ converge to zero. Moreover, $\vartheta_{w_{2}}$ and $\vartheta_{w_{3}}$ also go to zero as $e_{v_{s w_{j}, d q}} \rightarrow 0$. These results are helpful for the theoretical analysis in the next part.

When the closed-loop system (20) is in the steady-state condition, the following algebraic equations

$$
A \bar{z}+\bar{\vartheta}_{g}+\bar{\vartheta}_{w}=0
$$

must hold where the notation $(\bar{\cdot})$ denotes the steady-state value of the variable or vector $(\cdot)$.

Since $P_{g_{i}}^{o}=3 / 2\left(v_{s g_{i}} d i_{g_{i} d}^{o}+v_{s g_{i} q} i_{g_{i} q}^{o}\right), \bar{\vartheta}_{g}$ and $\bar{\vartheta}_{w}$ can be then expressed as:

$$
\begin{aligned}
& \bar{\vartheta}_{g}(i)=\left\{\begin{array}{l}
\frac{P_{g_{i}}^{0}+\frac{3}{2} k_{d_{i}} v_{s g_{i} d} u_{c g_{i}}^{o}}{C_{g_{i}} \bar{u}_{g_{i}}}-\frac{3 v_{s g_{i} d}}{2 C_{g_{i}}} k_{d_{i}}, i \in \mathcal{N} \\
0 . \text { otherwise }
\end{array}\right. \\
& \bar{\vartheta}_{w}(j+N)= \begin{cases}\frac{P_{w_{j}}^{o}}{C_{w_{j}} u_{c w_{j}}}, & j \in \mathcal{M} \\
0 . & \text { otherwise }\end{cases}
\end{aligned}
$$

where we introduce the new variable:

$$
P_{w_{j}}^{o}=3 / 2\left(v_{s w_{j} d}^{o} I_{w_{j} d}+v_{s w_{j} q}^{o} I_{w_{j} q}\right)
$$

Remark 7: From the algebraic equation (21), it can be seen that: a) Prescribed setpoints $(\cdot)^{o}$; b) DC grid topology; c) Droop gains $k_{d_{i}}$, determine the steady-state values of the system variables and the distribution of efforts for the power sharing in case of power imbalance. The control gains $k_{1 g_{i}, d q}$, $k_{2 g_{i}, d q}, k_{1 w_{j}, d q}, k_{2 w_{j}, d q}, k_{1, d q_{j}}$ and $k_{2, d q_{j}}$ have no effect on the steady state of the system. However, solving equation (21) is not easy and is not considered in this paper. More details about this issue can be referred in [9], [33].

To ensure the operating feasibility of the MTDC system, we assume that $\bar{i}_{g_{i} d q}, \bar{i}_{w_{j} d q}, \bar{u}_{c g_{i}}, \bar{u}_{c w_{j}}, \bar{u}_{c t_{h}}$ and $\bar{i}_{c_{k}}$ exist and belong to their respective safe operating domains. The equilibrium of the closed-loop system is denoted by $\bar{S}$.

Remark 8: In stead-state condition, From the droop law (17), we have:

$$
\bar{P}_{g_{i}}=P_{g_{i}}^{o}+K_{d_{i}}\left(u_{c g_{i}}^{o}-\bar{u}_{c g_{i}}\right)
$$

Consider that the MTDC system initially $\left(t=t_{0}\right)$ operates in a steady-state condition, if the production of the wind farms increases at the instant $t_{1}$, this makes the GVSCs absorb more power from the DC grid. When the MTDC system achieves a new steady state at $t_{2}$, we have $\bar{P}_{g_{i}}\left(t_{2}\right)<\bar{P}_{g_{i}}\left(t_{0}\right)$ (for example, $\bar{P}_{g_{i}}\left(t_{0}\right)=-100 \mathrm{MW}$ and $\left.\bar{P}_{g_{i}}\left(t_{2}\right)=-200 \mathrm{MW}\right)$. According to (23), we can deduce that the DC voltage also rises to a new level and that $\bar{u}_{g_{i}}\left(t_{2}\right)>\bar{u}_{g_{i}}\left(t_{0}\right)$. It can be summarized that $u_{c g_{i}}$ will rise if more power is injected into the DC grid, and vice versa. This statement will be illustrated by numerical simulations in Section V.

\section{B. Multi-time-scale dynamics}

In this part, a theoretical analysis to describe the dynamic separation in time-scales is carried out. We present that two different dynamics are created by the designed control algorithm in Section III. In particular, the two time-scales are quantified by the fast control gains $k_{1 g_{i}, d q}, k_{2 g_{i}, d q}, k_{1 w_{j}, d q}$ and $k_{2 w_{j}, d q}$.

In order to make the analysis convenient, we perform a change of variables. Then, the first eight equations of the closed-loop system (20) can be rewritten as:

$$
\begin{aligned}
\frac{d e_{I i_{g_{i} d}}}{d t} & =e_{i_{g_{i} d}} \\
\frac{d e_{i_{i} d}}{d t} & =-k_{1 g_{i} d} e_{i_{g_{i} d}}-k_{2 g_{i} d} e_{I i_{g_{i} d}}-\frac{d i_{g_{i} d}^{*}}{d t} \\
\frac{d e_{I i_{g_{i} q}}^{*}}{d t} & =e_{i_{g_{i} q}} \\
\frac{d e_{i_{g_{i}} q}}{d t} & =-k_{1 g_{i} q} e_{i_{g_{i} q}}-k_{2 g_{i} q} e_{I i_{g_{i} q}}-\frac{d i_{g_{i} q}^{*}}{d t} \\
\frac{d e_{I i_{w_{j}}}}{d t} & =e_{i_{w_{j} d}} \\
\frac{d e_{i_{w_{j} d}}}{d t} & =-k_{1 w_{j} d} e_{i_{w_{j} d}}-k_{2 w_{j} d} e_{I i_{w_{j} d}}-\frac{d i_{w_{j} d}^{*}}{d t} \\
\frac{d e_{I i_{w_{j} q} q}^{*}}{d t} & =e_{i_{w_{j} q}} \\
\frac{d e_{i_{w_{j}} q}}{d t} & =-k_{1 w_{j} q} e_{i_{w_{j} q}}-k_{2 w_{j} q} e_{I i_{w_{j} q}}-\frac{d i_{w_{j} q}^{*}}{d t}
\end{aligned}
$$

This shifts the quasi-steady states of the $d q$ currents to the origin.

Denote $k_{1}=\min \left(k_{1 g_{i} d}, k_{1 g_{i} q}, k_{1 w_{j} d}, k_{1 w_{j} q}\right)$ and then introduce a new variable $\epsilon$ satisfying:

$$
\epsilon k_{1}=1
$$

The subsystem (24) can be rewritten as:

$$
\begin{aligned}
& \frac{d e_{I i_{g_{i}} d}}{d t}=e_{i_{g_{i} d}} \\
& \epsilon \frac{d e_{g_{i} d}}{d t}=-\bar{k}_{1 g_{i} d} e_{i_{g_{i} d}}-\epsilon k_{2 g_{i} d} e_{I i_{g_{i} d}}-\epsilon \frac{d i_{g_{i} d}^{*}}{d t} \\
& \frac{d e^{I i_{g_{i} q}}}{d t}=e_{i_{g_{i} q}} \\
& \epsilon \frac{d e_{i_{i} q}}{d t}=-\bar{k}_{1 g_{i} q} e_{i_{g_{i} q}}-\epsilon k_{2 g_{i} q} e_{I i_{g_{i} q}}-\epsilon \frac{d i_{i_{i} q}^{*}}{d t} \\
& \frac{d e_{I i} w_{j} d}{d t}=e_{i_{w_{j} d}} \\
& \epsilon \frac{d e_{w_{w_{j} d}}}{d t}=-\bar{k}_{1 w_{j} d} e_{i_{w_{j} d}}-\epsilon k_{2 w_{j} d} e_{I i_{w_{j} d}}-\epsilon \frac{d i_{w_{j} d}^{*}}{d t} \\
& \frac{d e_{I i_{w_{j}} q}}{d t}=e_{i_{w_{j} q}} \\
& \epsilon \frac{d e_{i_{w_{j} q}}}{d t}=-\bar{k}_{1 w_{j} q} e_{i_{w_{j} q}}-\epsilon k_{2 w_{j} q} e_{I i_{w_{j} q}}-\epsilon \frac{d i_{w_{j} q}^{*}}{d t}
\end{aligned}
$$

where

$$
\begin{gathered}
\bar{k}_{1 g_{i} d}=\epsilon k_{1 g_{i} d} \geq 1 ; \bar{k}_{1 g_{i} q}=\epsilon k_{1 g_{i} q} \geq 1 \\
\bar{k}_{1 w_{j} d}=\epsilon k_{1 w_{j} d} \geq 1 ; \bar{k}_{1 w_{j} q}=\epsilon k_{1 w_{j} q} \geq 1
\end{gathered}
$$

We define a new time variable $\tau$ as:

$$
\epsilon \frac{d y}{d t}=\frac{d y}{d \tau}
$$


Substituting (26) into (25) and then setting $\epsilon=0$, we obtain the following autonomous system:

$$
\begin{aligned}
& \frac{d e_{i_{g_{i}} d}}{d \tau}=-\bar{k}_{1 g_{i} d} e_{i_{g_{i} d}} \\
& \frac{d e_{i_{i} q}}{d \tau}=-\bar{k}_{1 g_{i} q} e_{i_{g_{i} q}} \\
& \frac{d e_{i_{w_{j}}}}{d \tau}=-\bar{k}_{1 w_{j} d} e_{i_{w_{j} d}} \\
& \frac{d e_{i_{w_{j} q}}}{d \tau}=-\bar{k}_{1 w_{j} q} e_{i_{w_{j} q}}
\end{aligned}
$$

which is called the boundary-layer model of the system (20). When $\epsilon \rightarrow 0$, it seems that the time variable $t$ and the slow state variables are frozen at their initial values. By using Lyapunov analysis, the origin of the boundary system (27) is verified as exponentially stable. When the $d q$ currents quickly converge to their reference trajectories during the initial interval, the full-scale system (20) is degenerated into the following reduced model:

$$
\begin{aligned}
\frac{d e_{I v_{s w_{j} d}}}{d t} & =e_{v_{s w_{j} d}} \\
\frac{d v_{s w_{j} d}}{d t} & =-k_{1 d_{j}} e_{v_{s w_{j} d}}-k_{2 d_{j}} e_{I v_{s w_{j} d}} \\
\frac{d e_{I v_{s w_{j} q}}}{d t} & =e_{v_{s w_{j} q}} \\
\frac{d v_{s w_{j} q}}{d t} & =-k_{1 q_{j}} e_{v_{s w_{j} q}}-k_{2 q_{j}} e_{I v_{s w_{j} q}} \\
\frac{d z}{d t} & =A z+\vartheta_{g}^{\prime}+\vartheta_{w}^{\prime}
\end{aligned}
$$

where $\vartheta_{g}^{\prime}=\vartheta_{g_{1}}+\vartheta_{g_{2}}$ and $\vartheta_{w}^{\prime}=\vartheta_{w_{1}}+\vartheta_{w_{2}}+\vartheta_{w_{3}}$. In addition, the solution of the reduced model (28) is denoted by $(\cdot)^{r e}$. Now, we can state the first result of this paper.

Theorem 1: Consider the system (20) where all state variables are restrict to their respective arbitrary operating domains for $t \in\left[t_{0}, t_{1}\right]$. There exists a positive constant $\epsilon^{*}$ such that for all $0<\epsilon=1 / k_{1}<\epsilon^{*}$, the system (20) has a unique solution on $\left[t_{0}, t_{1}\right]$, and the trajectories of the state variables can be approximated by:

$$
\begin{aligned}
i_{g_{i}, d q}-i_{g_{i}, d q}^{*}\left(z^{r e}\right)-\widehat{e}_{i_{g_{i}, d q}} & =O(\epsilon) \\
i_{w_{j}, d q}-i_{w_{j}, d q}^{*}\left(z^{r e}, v_{s w_{j}, d q}^{r e}\right)-\widehat{e}_{i_{w_{j}, d q}} & =O(\epsilon) \\
v_{s w_{j}, d q}-v_{s w_{j}, d q}^{r e} & =O(\epsilon) \\
z-z^{r e} & =O(\epsilon)
\end{aligned}
$$

held uniformly for $t \in\left[t_{0}, t_{1}\right]$ where $\hat{e}_{i_{g_{i}, d q}}$ and $\hat{e}_{i_{w_{j}, d q}}$ are the solution of the boundary-layer model (27)

$$
\begin{aligned}
\widehat{e}_{i_{g_{i}, d q}} & =e_{i_{g_{i}, d q}}\left(t_{0}\right) \exp \left(-\bar{k}_{1 g_{i}, d q} \tau\right) \\
\widehat{e}_{i_{w_{j}, d q}} & =e_{i_{w_{j}, d q}}\left(t_{0}\right) \exp \left(-\bar{k}_{1 w_{j}, d q} \tau\right)
\end{aligned}
$$

Proof: It is obvious that the origin of the boundarylayer model (27) is globally exponentially stable. Besides, the reduced model (28) has a unique solution. Therefore, the approximations (29) can be obtained by direct application of Theorem 11.1 in [36].

Remark 9: In terms of (29), the $d q$ currents exhibit a twotime-scale behavior by presenting a slow and a fast transients. It is the $d q$ current tracking errors, i.e. $\widehat{e}_{i_{g_{i}, d q}}$ and $\widehat{e}_{i_{w_{j}, d q}}$, that characterize the part of fast dynamics. It is shown that $i_{(\cdot), d q}$ start with a fast transient which exactly corresponds to the solution of boundary-layer model (27) during the initial interval. After the exponential decay of the fast transients $\widehat{e}_{i_{g_{i}, d q}}\left(\widehat{e}_{i_{w_{j}, d q}}\right), i_{(\cdot), d q}$ remain close to their respective manifolds $i_{(\cdot), d q}^{*}$ in the future time. Since the fast transient only significantly appears on $i_{(\cdot), d q}$, we call the $d q$ currents the fast dynamic variables while $v_{s w_{j}, d q}$ and $z$ are the slow dynamic variables.

Remark 10: As expressed in (30), the control gains $k_{1 g_{i}, d q}$, $k_{2 g_{i}, d q}, k_{1 w_{j}, d q}$ and $k_{2 w_{j}, d q}$ play a major role in regulating the fast transient performance and hence we call them the fast control gains. The control gains $k_{1, d q_{j}}, k_{2, d q_{j}}$ and $k_{d_{i}}$, dominate the slow transient performance, and they are consequently called the slow control gains.

\section{Stability analysis}

For the sake of simplicity, we introduce a new variable

$$
e_{z}=z-\bar{z}
$$

Now, the closed-loop system (20) becomes:

$$
\begin{aligned}
\frac{d e_{I i_{g_{i}} d}}{d t} & =e_{i_{g_{i} d}} \\
\frac{d e_{i_{g_{i}}}}{d t} & =-k_{1 g_{i} d} e_{i_{g_{i} d}}-k_{2 g_{i} d} e_{I i_{g_{i} d}}-\frac{d i_{g_{i} d}^{*}}{d t} \\
\frac{d e_{I i_{i} q}}{d t} & =e_{i_{g_{i} q}} \\
\frac{d e_{i_{i} q}}{d t} & =-k_{1 g_{i} q} e_{i_{g_{i} q}}-k_{2 g_{i} q} e_{I i_{g_{i} q}}-\frac{d i_{g_{i} q}^{*}}{d t} \\
\frac{d e_{I i_{w_{j}}}}{d t} & =e_{i_{w_{j} d}} \\
\frac{d e_{i_{w_{j}} d}}{d t} & =-k_{1 w_{j} d} e_{i_{w_{j} d}}-k_{2 w_{j} d} e_{I i_{w_{j} d}}-\frac{d i_{w_{j} d}^{*}}{d t} \\
\frac{d e_{I i_{w_{j}} q}}{d t} & =e_{i_{w_{j} q}} \\
\frac{d e_{i_{w_{j}} q}}{d t} & =-k_{1 w_{j} q} e_{i_{w_{j} q}}-k_{2 w_{j} q} e_{I i_{w_{j} q}}-\frac{d i_{w_{j} q}^{*}}{d t} \\
\frac{d e_{I v_{s w_{j} d}}}{d t} & =e_{v_{s w_{j} d}} \\
\frac{d e_{v_{s w_{j} d}}}{d t} & =-k_{1 d_{j}} e_{v_{s w_{j} d}}-k_{2 d_{j}} e_{I v_{s w_{j} d}}-\frac{e_{i_{w_{j} d}}}{C_{f w_{j}}} \\
\frac{d e_{I v_{s w_{j} q}}}{d t} & =e_{v_{s w_{j} q}} \\
\frac{d e_{v_{s w_{j} q}}}{d t} & =-k_{1 q_{j}} e_{v_{s w_{j} q}}-k_{2 q_{j}} e_{I v_{s w_{j} q}}-\frac{e_{i_{w_{j} q} q}}{C_{f w_{j}}} \\
\frac{d e_{z}}{d t} & =A e_{z}+A \bar{z}+\vartheta_{g}+\vartheta_{w}
\end{aligned}
$$

where the equilibrium of the original closed-loop system, $\bar{S}$, is shifted to the origin. Moreover, there exist operating domains for these new state variables that correspond to the arbitrary safe operating regions mentioned in Remark 2. We call (32) the error system of the closed-loop system. In terms of those tracking errors, the varying reference trajectories (16) can be expressed by:

$$
\begin{aligned}
i_{w_{j} d}^{*}= & C_{f w_{j}} \omega_{w_{j}}\left(v_{s w_{j} q}^{o}+e_{v_{s w_{j} q}}\right)+I_{w_{j} d} \\
& +k_{1 d_{j}} e_{v_{s w_{j} d}}+k_{2 d_{j}} e_{I v_{s w_{j} d}} \\
i_{w_{j} q}^{*}= & -C_{f w_{j}} \omega_{w_{j}}\left(v_{s w_{j} d}^{o}+e_{v_{s w_{j} d}}\right)+I_{w_{j} q} \\
& +k_{1 q_{j}} e_{v_{s w_{j} q}}+k_{2 q_{j}} e_{I v_{s w_{j} q}}
\end{aligned}
$$

Since $I_{w_{j}, d q}$ are considered constant or slowly varying and then we take $\frac{d I_{w_{j}, d q}}{d t}=0$, the derivatives of the $d q$ references can be expressed as:

$$
\begin{aligned}
& \frac{d i_{g_{i} d}^{*}}{d t}=-k_{d_{i}} \frac{d e_{u_{g_{i}}}}{d t}, \quad \frac{d i_{g_{i} q}^{*}}{d t}=0 \\
& \frac{d i_{w_{j} d}^{*}}{d t}=C_{f w_{j}} \omega_{w_{j}} \frac{d e_{v_{s w_{j} q}}}{d t}+k_{1 d_{j}} \frac{d e_{v_{s w_{j} d}}}{d t}+k_{2 d_{j}} e_{v_{s w_{j} d}} \\
& \frac{d i_{w_{j} q}^{*}}{d t}=-C_{f w_{j}} \omega_{w_{j}} \frac{d e_{v_{s w_{j} d}}}{d t}+k_{1 q_{j}} \frac{d e_{v_{s w_{j} q}}}{d t}+k_{2 q_{j}} e_{v_{s w_{j} q}}
\end{aligned}
$$


Remark 11: Note that the asymptotic stability of the origin of the error system (32) would imply the asymptotic stability of the equilibrium $\bar{S}$ of the original closed-loop system (20). Hence, we investigate the stability of the error system (32) instead of the original closed-loop system (20).

If $e_{i_{g_{i} d q}}$ and $e_{i_{w_{j} d q}}$ converge to zero, a new reduced model is deduced from the error system as:

$$
\begin{aligned}
\frac{d e_{I v_{s w_{j} d}}}{d t} & =e_{v_{s w_{j} d}} \\
\frac{d e_{v_{s w_{j}}}}{d t} & =-k_{1 d_{j}} e_{v_{s w_{j} d}}-k_{2 d_{j}} e_{I v_{s w_{j} d}} \\
\frac{d e_{I v_{s w_{j} q}}}{d t} & =e_{v_{s w_{j} q}} \\
\frac{d e_{v_{s w_{j} q}}}{d t} & =-k_{1 q_{j}} e_{v_{s w_{j} q}}-k_{2 q_{j}} e_{I v_{s w_{j} q}} \\
\frac{d e_{z}}{d t} & =A e_{z}+A \bar{z}+\vartheta_{g}^{\prime}+\vartheta_{w}^{\prime}
\end{aligned}
$$

where $\vartheta_{g}^{\prime}=\vartheta_{g_{1}}+\vartheta_{g_{2}}$ and $\vartheta_{w}^{\prime}=\vartheta_{w_{1}}+\vartheta_{w_{2}}$. The reduced model (35) can be divided into two parts, the external dynamics represented by $e_{v_{s w_{j}, d q}}$ and $e_{I v_{s w_{j}, d q}}$ and the internal dynamics represented by $e_{z}$. As the external variables converge to zero, i.e. $e_{v_{s w_{j}, d q}} \rightarrow 0$ and $e_{I v_{s w_{j}, d q}} \rightarrow 0$, the behavior of the reduced model (35) is governed by the internal subsystem:

$$
\frac{d e_{z}}{d t}=A e_{z}+A \bar{z}+\vartheta_{g}^{\prime}+\vartheta_{w}^{\prime} \triangleq f_{z e r o}
$$

We call (36) the zero dynamics of the reduced model (35).

Remark 12: In the reduced model (35), we remark that the dynamics of the external variables are controlled by $k_{1, d q_{j}}$ and $k_{2, d q_{j}}$ while $k_{d_{i}}$ have a great effect on the dynamics of the internal variables (or the dynamics of the DC grid). Recalling Remark 10, it can be summarized that the control gains have different impacts on the system performance.

Lemma 3: Consider the reduced model (35). Fix positive control gains $k_{1,2 d_{j}}$ and $k_{1,2 q_{j}}, \forall j \in \mathcal{M}$. Select the droop gains $k_{d_{i}}$ that satisfy the following conditions:

- $\forall i \in \mathcal{N}, k_{d_{i}}$ is chosen such that:

$$
k_{d_{i}} \geq k_{d_{\text {min }}}
$$

where

$$
P_{g_{i}}^{o}+\frac{3}{2} v_{s g_{i} d} k_{d_{i \min }} u_{c g_{i}}^{o}=0
$$

- There exists at least one GVSC terminal whose droop gain satisfies:

$$
k_{d_{q}}>k_{d_{q \min }}
$$

where $q \in \mathcal{N}$.

Then, the origin of the reduced model (35) is locally asymptotically stable.

The following properties of complex matrix as referred in [37] is applied to prove the lemma.

Definition 1: A matrix $\Psi \in \mathbb{C}^{n \times n}$ is said to be positive definite if $\operatorname{Re}\left(x^{H} \Psi x\right)$ is positive for every non-zero column vector $x \in \mathbb{C}^{n \times 1}$. The set of positive definite matrix $\Psi$ is denoted as $P_{C}^{+}$.

Lemma 4: Matrix $\Psi \in \mathbb{C}^{n \times n}$ is positive definite if and only if its Hermitian part $\mathcal{H}(\Psi)$ is positive definite.

Lemma 5: If $\Psi \in P_{C}^{+}$, then $\Psi$ is invertible and $\Psi^{-1} \in P_{C}^{+}$. We now can prove Lemma 3 as follows.
Proof: For the system described by (35), if the zero dynamics (36) is locally asymptotically stable, then the origin of the system (35) is locally asymptotically stable [38]. Therefore, to establish the claim, we first study the stability of the zero dynamics (36). This is done by linearizing the zero dynamics (36) around the origin. The Jacobian matrix $\left.\left[\frac{\partial f_{z e r_{0}}}{\partial e_{z}}\right]\right|_{e_{z}=0}$ is expressed by:

$$
\left.\Phi \triangleq\left[\frac{\partial f_{\text {zero }}}{\partial e_{z}}\right]\right|_{e_{z}=0}=\left[\begin{array}{cc}
D & C^{-1} H \\
-L^{-1} H^{T} & -L^{-1} R
\end{array}\right]
$$

where $D=\operatorname{diag}\left(d_{k}\right), k=1, \cdots, N+M+P$, is a diagonal matrix given by:

$$
\left\{\begin{aligned}
d_{i} & =-\frac{P_{g_{i}}^{o}+\frac{3}{2} v_{s g_{i} d} k_{d_{i}} u_{g_{i}}^{o}}{C_{g_{i}} \bar{u}_{g_{i}}^{2}}, & & i \in \mathcal{N} \\
d_{(j+N)} & =-\frac{P_{w_{j}}^{o}}{C_{w_{j}} \bar{u}_{w_{j}}^{2}}, & & j \in \mathcal{M} \\
d_{(h+N+M)} & =0 . & & h \in \mathcal{P}
\end{aligned}\right.
$$

Let us assume that there exists a particular eigenvalue of $\Phi$ denoted by $\lambda=\alpha+j \beta \in \mathbb{C}$, whose real part is non-negative, i.e. $\alpha \geq 0$. Then, $\lambda$ satisfies:

$$
\operatorname{det}(\lambda I-\Phi)=0
$$

Alternatively, it can be expressed as:

$$
\operatorname{det}\left(\left[\begin{array}{cc}
\lambda I-D & -C^{-1} H \\
L^{-1} H^{T} & \lambda I+L^{-1} R
\end{array}\right]\right)=0
$$

We define $\Phi_{1} \triangleq \lambda I+L^{-1} R=\Lambda_{1}+j \Lambda_{2}$ where $\Lambda_{1,2}$ are expressed by:

$$
\begin{aligned}
& \Lambda_{1}=\operatorname{diag}\left(\alpha+\frac{R_{c_{1}}}{L_{c_{1}}}, \cdots, \alpha+\frac{R_{c_{L}}}{L_{c_{L}}}\right) \in \mathbb{R}^{L \times L} \\
& \Lambda_{2}=\operatorname{diag}(\beta, \cdots, \beta) \in \mathbb{R}^{L \times L}
\end{aligned}
$$

Since the Hermitian part of $\Phi_{1}$ is equal to $\mathcal{H}\left(\Phi_{1}\right)=\Lambda_{1}$, which is positive definite, the complex matrix $\Phi_{1}$ is also positive definite (Lemma 4). Consequently, $\Phi_{1}$ must be invertible (Lemma 5) and then, Eq. (40) becomes:

$$
\begin{aligned}
& \operatorname{det}\left(\left[\begin{array}{cc}
\lambda I-D & -C^{-1} H \\
L^{-1} H^{T} & \lambda I+L^{-1} R
\end{array}\right]\right) \\
= & \operatorname{det}\left(\lambda I-D+C^{-1} H \Phi_{1}^{-1} L^{-1} H^{T}\right) \operatorname{det}\left(\Phi_{1}\right) \\
= & \operatorname{det}\left(\lambda C-C D+H(\lambda L+R)^{-1} H^{T}\right) \operatorname{det}\left(\Phi_{1}\right) \operatorname{det}\left(C^{-1}\right)
\end{aligned}
$$

Again, we define:

$$
\begin{aligned}
\Phi_{2} & =\lambda C-C D+H(\lambda L+R)^{-1} H^{T} \\
\lambda C-C D & =\Lambda_{3}+j \Lambda_{4} \\
(\lambda L+R)^{-1} & =\Lambda_{5}+j \Lambda_{6}
\end{aligned}
$$


with the notations:

$$
\begin{aligned}
& \Lambda_{3}=\alpha C-C D \triangleq \operatorname{diag}\left(\sigma_{1}, \cdots, \sigma_{N+M+P}\right) \\
& \left\{\begin{array}{cc}
\sigma_{i}=C_{g_{i}}\left(\alpha+\frac{P_{g_{i}}^{o}+\frac{3}{2} v_{s_{i} d} k_{d_{i}} u_{g_{i}}^{o}}{C_{g_{i}} \bar{u}_{g_{i}}^{2}}\right), & i \in \mathcal{N} \\
\sigma_{(j+N)}=C_{w_{j}}\left(\alpha+\frac{P_{w_{j}}^{o}}{C_{w_{j}} \bar{u}_{w_{j}}^{2}}\right), & j \in \mathcal{M} \\
\sigma_{(h+N+M)}=C_{p_{h}} \alpha . & h \in \mathcal{P} \\
\Lambda_{4}=\beta C & \\
\Lambda_{5}=\operatorname{diag}\left(\frac{\alpha L_{c_{k}}+R_{c_{k}}}{\left(\alpha L_{c_{k}}+R_{c_{k}}\right)^{2} L_{c_{k}}\left(\beta L_{c_{k}}\right)^{2}}\right) \in \mathbb{R}^{L \times L} & \\
\Lambda_{6}=\operatorname{diag}\left(\frac{\left.-\beta L_{c_{k}}+R_{c_{k}}\right)^{2}+\left(\beta L_{c_{k}}\right)^{2}}{(\alpha)}\right) \in \mathbb{R}^{L \times L} &
\end{array}\right.
\end{aligned}
$$

Due to (38), we assume that $k_{d_{q}}>k_{d_{q}, \min }, q \in \mathcal{N}$, where

$$
P_{g_{q}}^{o}+\frac{3}{2} v_{s g_{q} d} k_{d_{q} \cdot \min } u_{g_{q}}^{o}=0
$$

Now, $\Phi_{2}$ can be rewritten as:

$$
\begin{aligned}
& \Phi_{2}=\Phi_{3}+j\left(\Lambda_{4}+H \Lambda_{6} H^{T}\right) \\
& \Phi_{3}=\Lambda_{3}^{q}+\Lambda_{3}^{q^{\prime}}+H_{f} H_{f}^{T}
\end{aligned}
$$

where $\Lambda_{3}^{q}$ is a diagonal matrix whose $q^{\text {th }}$ element on the main diagonal is $\sigma_{q}$ and other elements are zero. $\Lambda_{3}^{q^{\prime}}$ and $H_{f}$ are given by $\Lambda_{3}^{q^{\prime}}=\Lambda_{3}-\Lambda_{3}^{q}$ and $H_{f}=H \Lambda_{5}^{\frac{1}{2}}$.

Let us define $\Phi_{4} \triangleq \Lambda_{3}^{q}+H_{f} H_{f}^{T}$ and then the determinant of $\Phi_{4}$ can be calculated as:

$$
\operatorname{det}\left(\Phi_{4}\right)=\sigma_{q} \operatorname{det}\left(\mathcal{R}\left(H_{f}\right)_{q} \mathcal{R}\left(H_{f}\right)_{q}^{T}\right)+\operatorname{det}\left(H_{f} H_{f}^{T}\right)
$$

Since $\Lambda_{5}^{\frac{1}{2}}$ is a full rank matrix, i.e. $\operatorname{rank}\left(\Lambda_{5}^{\frac{1}{2}}\right)=L$, then $\operatorname{rank}\left(H_{f} H_{f}^{T}\right)=\operatorname{rank}\left(H_{f}\right)=\operatorname{rank}(H)=N+M+P-1$ and as a result, $\operatorname{det}\left(H_{f} H_{f}^{T}\right)=0$. Similar to Lemma 2 , by removing any one row from $H_{f}$, such as $H_{f}(j,:), j \in \mathcal{T}$, the rank of the reduced matrix $\mathcal{R}\left(H_{f}\right)_{j}$ is $N+M+P-1$. Consequently, $\mathcal{R}\left(H_{f}\right)_{q} \mathcal{R}\left(H_{f}\right)_{q}^{T}$ is invertible and positive definite. Since $k_{d_{q}}$ satisfies (42), we get $\sigma_{q}>0$ and then, $\operatorname{det}\left(\Phi_{4}\right)=\sigma_{q} \operatorname{det}\left(\mathcal{R}\left(H_{f}\right)_{q} \mathcal{R}\left(H_{f}\right)_{q}^{T}\right)>0$. On the other hand, $\Phi_{4}$ and $\Lambda_{3}^{q}$ are semi-positive definite because of (37) and (38) and hence, $\Phi_{4}$ is positive definite. Recalling that $\Phi_{3}=\Phi_{4}+\Lambda_{3}^{q^{\prime}}$, it turns out that, $\Phi_{3}$ is also positive definite. As presented in (43), we know that $\mathcal{H}\left(\Phi_{2}\right)=\Phi_{3}$ and then, according to Lemma 4 and Lemma $5, \Phi_{2}$ is positive definite and invertible. Therefore, the following result is obtained:

$$
\operatorname{det}(\lambda I-\Phi)=\operatorname{det}\left(\Phi_{2}\right) \operatorname{det}\left(\Phi_{1}\right) \operatorname{det}\left(C^{-1}\right) \neq 0
$$

for $\alpha \geq 0$. This leads to a contradiction to (40). Thus, all eigenvalues of the Jacobian matrix $\Phi$ must have negative real part, i.e. $\alpha<0$. Hence, $\Phi$ is a Hurwitz matrix. As a result, the origin of the zero dynamics (36) is locally asymptotically stable. Thus, the reduced model (35) is also locally asymptotically stable. The proof is completed.

Now, we can introduce another result of this paper.

Theorem 2: Consider the MTDC system modeled by (1), (3), (4) and (7) with the control strategy (12), (16), (18) and (19). Select the droop gains that satisfy the conditions (37) and (38) in Lemma 3. Then, there exist control gains $k_{1 g_{i} d}, k_{1 g_{i} q}$, $k_{1 w_{j} d}, k_{1 w_{j} q}, k_{1,2 d_{j}}$ and $k_{1,2 q_{j}}$ such that the equilibrium $\vec{S}$ of the closed loop system (20) is locally asymptotically stable.
Thereby, the proposed control strategy can stabilize the MTDC system.

Proof: As previously mentioned in Remark 11, to study the stability of the origin of the error system (32) is equivalent to investigate the stability of the equilibrium $\bar{S}$ of the closedloop system (20). So we still focus on the stability property of the error system (32).

We rewrite the last equation in (32) in the following special form:

$$
\frac{d e_{z}}{d t}=f_{z e r o}+\vartheta_{g_{3}}+\vartheta_{w_{2}}+\vartheta_{w_{3}}+\vartheta_{w_{4}}
$$

According to Taylor's theorem, $f_{z e r o}$ can be expressed by:

$$
f_{\text {zero }}=\Phi \cdot e_{z}+\tilde{f}_{z e r o} \cdot e_{z}
$$

where $\tilde{f}_{\text {zero }}$ satisfies:

$$
\lim _{\left\|e_{z}\right\| \rightarrow 0}\left\|\tilde{f}_{z e r o}\right\|=0
$$

It means that, for any given $\alpha_{0}$, there exist a region $\mathbb{B}_{e_{z}}$ such that:

$$
\left\|\tilde{f}_{z e r o}\right\| \leq \alpha_{0}
$$

for all $e_{z} \in \mathbb{B}_{e_{z}}$. In addition, since $\Phi$ is a Hurwitz matrix, for any positive definite matrix $G_{e_{z}}$, there exists a positive definite matrix $F_{e_{z}}$ such that:

$$
F_{e_{z}} \Phi+\Phi^{T} F_{e_{z}}=-G_{e_{z}}
$$

To simplify the notations, we introduce the following variables:

$$
\begin{aligned}
e_{i_{g, d q}} & \triangleq\left[\begin{array}{lllll}
e_{i_{g_{1}, d q}} & e_{I i_{g_{1}, d q}} & \cdots & e_{i_{g_{N}, d q}} & e_{I i_{g_{N}, d q}}
\end{array}\right]^{T} \\
e_{i_{w, d q}} & \triangleq\left[\begin{array}{lllll}
e_{i_{w_{1}, d q}} & e_{I i_{w_{1}, d q}} & \cdots & e_{i_{w_{M}, d q}} & e_{I i_{w_{M}, d q},{ }}
\end{array}\right]^{T} \\
e_{v_{w, d q}} & \triangleq\left[\begin{array}{lllll}
e_{v_{s w_{1}, d q}} & e_{I v_{s w_{1}, d q}} & \cdots & e_{v_{s w_{M}, d q}} & e_{I v_{s w_{M}, d q}}
\end{array}\right]^{T}
\end{aligned}
$$

Consider a Lyapunov function $W=d W_{1}+(1-d) W_{2}$ where $d \in(0,1) . W_{1}$ and $W_{2}$ are designed as:

$$
\begin{aligned}
W_{1}= & \frac{1}{2}\left(e_{i_{g d}}^{T} F_{i_{g d}} e_{i_{g d}}+e_{i_{g q}}^{T} F_{i_{g q}} e_{i_{g q}}\right. \\
& \left.+e_{i_{w d}}^{T} F_{i_{w d}} e_{i_{w d}}+e_{i_{w q}}^{T} F_{i_{w q}} e_{i_{w q}}\right)
\end{aligned}
$$

and

$$
W_{2}=\frac{1}{2}\left(e_{v_{w d}}^{T} F_{v_{w d}} e_{v_{w d}}+e_{v_{w q}}^{T} F_{v_{w q}} e_{v_{w q}}+e_{z}^{T} F_{e_{z}} e_{z}\right)
$$

where

$$
\begin{aligned}
& F_{i_{g, d q}}=\operatorname{diag}\left(F_{i_{g, d q}}^{i}\right) ; F_{i_{g, d q}}^{i}=\left[\begin{array}{cc}
1 & 0 \\
0 & k_{2 g_{i}, d q}
\end{array}\right] \\
& F_{i_{w, d q}}=\operatorname{diag}\left(F_{i_{w, d q}}^{j}\right) ; F_{i_{w, d q}}^{j}=\left[\begin{array}{cc}
1 & 0 \\
0 & k_{2 w_{j}, d q}
\end{array}\right] \\
& F_{v_{w, d q}}=\operatorname{diag}\left(F_{v_{w, d q}}^{j}\right) \\
& F_{v_{w, d q}}^{j}=\left[\begin{array}{cc}
1+\frac{1}{k_{1, d q_{j}}} & 1 \\
1 & k_{1, d q_{j}}+k_{2, d q_{j}}+\frac{k_{2, d q_{j}}}{k_{1, d q_{j}}}
\end{array}\right]
\end{aligned}
$$


It is clear that $F_{i_{g, d q}}, F_{i_{w, d q}}$ and $F_{v_{w, d q}}$ are positive definite. Then, the derivative of $W$ along the trajectories (32) can be calculated by $\frac{d W}{d t}=D_{1}+D_{2}$ where:

$$
\begin{aligned}
& D_{1}=\Gamma_{1}+\Gamma_{2} \\
& D_{2}=e_{i_{g d}}^{T} F_{i_{g d}} \frac{d \eta_{i_{g d}}}{d t}+e_{i_{g q}}^{T} F_{i_{g q}} \frac{d \eta_{i_{g q}}}{d t}+e_{i_{w d}}^{T} F_{i_{w d}} \frac{d \eta_{i_{w d}}}{d t} \\
& +e_{i_{w q}}^{T} F_{i_{w q}} \frac{d \eta_{i_{w q}}}{d t}+e_{v_{w q}}^{T} F_{v_{w q}} \eta_{v_{w q}}+e_{v_{w d}}^{T} F_{v_{w d}} \eta_{v_{w d}} \\
& +e_{z}^{T} F_{e_{z}}\left(\tilde{f}_{z e r o} \cdot e_{z}+\vartheta_{g_{3}}+\vartheta_{w_{2}}+\vartheta_{w_{3}}+\vartheta_{w_{4}}\right)
\end{aligned}
$$

with

$$
\begin{aligned}
\eta_{i_{g, d q}} & \triangleq\left[\begin{array}{lllll}
i_{g_{1}, d q}^{*} & 0 & \ldots & i_{g_{N}, d q}^{*} & 0
\end{array}\right]^{T} \\
\eta_{i_{w, d q}} & \triangleq\left[\begin{array}{lllll}
i_{w_{1}, d q}^{*} & 0 & \ldots & i_{w_{M}, d q}^{*} & 0
\end{array}\right]^{T} \\
\eta_{v_{w, d q}} & \triangleq\left[\begin{array}{lllll}
\frac{e_{i_{w_{1}, d q}}}{C_{f w_{1}}} & 0 & \ldots & \frac{e_{i_{w_{N}}, d q}}{C_{f w_{N}}} & 0
\end{array}\right]^{T}
\end{aligned}
$$

and the other expressions are given by:

$$
\begin{aligned}
\Gamma_{1} & =-\sum_{j=1}^{M}\left(k_{1 d_{j}} e_{v_{s w_{j} d}}^{2}+k_{2 d_{j}} e_{I v_{w_{j} d}}^{2}+k_{1 q_{j}} e_{v_{s w_{j} q}}^{2}\right. \\
& \left.+k_{2 q_{j}} e_{I v_{w_{j} q}}^{2}\right)-e_{z}^{T} G_{e_{z}} e_{z} \\
\Gamma_{2} & =-\sum_{i=1}^{N}\left(k_{1 g_{i} d} e_{i_{g_{i}}}^{2}+k_{1 g_{i} q} e_{i_{g_{i} q}}^{2}\right) \\
& -\sum_{j=1}^{M}\left(k_{1 w_{j} d} e_{i_{w_{j}}}^{2}+k_{1 w_{j} q} e_{i_{w_{j} q}}^{2}\right) \\
G_{i_{g, d q}} & =\operatorname{diag}\left(G i_{g, d q}^{i}\right), G_{i_{g, d q}}^{i}=\left[\begin{array}{cc}
k_{1 g_{i}, d q} & 0 \\
0 & 0
\end{array}\right] \\
G_{i_{w, d q}} & =\operatorname{diag}\left(G_{i_{w, d q}}^{j}\right), G_{i_{w, d q}}^{j}=\left[\begin{array}{cc}
k_{1 w_{j}, d q} & 0 \\
0 & 0
\end{array}\right] \\
G_{v_{w, d q}} & =\operatorname{diag}\left(G_{v_{w, d q}}^{j}\right), G_{v_{w, d q}}^{j}=\left[\begin{array}{cc}
k_{1, d q_{j}} & 0 \\
0 & k_{2, d q_{j}}
\end{array}\right]
\end{aligned}
$$

Since the $d q$ current reference trajectories only depend on the slow dynamics state variables, we then have:

$$
\begin{aligned}
\frac{d \eta_{i_{g d}}}{d t} & =\frac{d \eta_{i_{g d}}}{d e_{z}} \frac{d e_{z}}{d t}, \frac{d \eta_{i_{g q}}}{d t}=\frac{d \eta_{i_{g q}}}{d e_{z}} \frac{d e_{z}}{d t} \\
\frac{d \eta_{i_{w d}}}{d t} & =\frac{d \eta_{i_{w d}}}{d e_{v_{w d}}} \frac{d e_{v_{w d}}}{d t}+\frac{d \eta_{i_{w d}}}{d e_{v_{w q}}} \frac{d e_{v_{w q}}}{d t}+\frac{d \eta_{i_{w d}}}{d e_{z}} \frac{d e_{z}}{d t} \\
\frac{d \eta_{i_{w q}}}{d t} & =\frac{d \eta_{i_{w q}}}{d e_{v_{w d}}} \frac{d e_{v_{w d}}}{d t}+\frac{d \eta_{i_{w q}}}{d e_{v_{w q}}} \frac{d e_{v_{w q}}}{d t}+\frac{d \eta_{i_{w q}}}{d e_{z}} \frac{d e_{z}}{d t}
\end{aligned}
$$

From the aforementioned sections, we know that:

$$
\begin{array}{r}
\left\|\vartheta_{g_{3}}\right\| \rightarrow 0 \text { as }\left\|e_{i_{g d}}\right\| \rightarrow 0 \text { and }\left\|e_{i_{g q}}\right\| \rightarrow 0 \\
\left\|\vartheta_{w_{4}}\right\| \rightarrow 0 \text { as }\left\|e_{i_{w d}}\right\| \rightarrow 0 \text { and }\left\|e_{i_{w q}}\right\| \rightarrow 0 \\
\left\|\vartheta_{w_{2}}\right\| \rightarrow 0,\left\|\vartheta_{w_{3}}\right\| \rightarrow 0 \text { as }\left\|e_{v_{w d}}\right\| \rightarrow 0 \text { and }\left\|e_{v_{w q}}\right\| \rightarrow 0
\end{array}
$$

and then there exist positive $\beta_{k}, k=1, \cdots, 8$ and convex regions $\mathbb{B}_{e_{i_{g}, d q}}, \mathbb{B}_{e_{i_{w}, d q}}, \mathbb{B}_{e_{v_{w}, d q}}$ such that:

$$
\begin{aligned}
& \left\|\vartheta_{g_{3}}\right\| \leq \beta_{1}\left\|e_{i_{g d}}\right\|+\beta_{2}\left\|e_{i_{g q}}\right\| \\
& \left\|\vartheta_{w_{4}}\right\| \leq \beta_{3}\left\|e_{i_{w d}}\right\|+\beta_{4}\left\|e_{i_{w q}}\right\| \\
& \left\|\vartheta_{w_{2}}\right\| \leq \beta_{5}\left\|e_{v_{w d}}\right\|+\beta_{6}\left\|e_{v_{w q}}\right\| \\
& \left\|\vartheta_{w_{3}}\right\| \leq \beta_{7}\left\|e_{v_{w d}}\right\|+\beta_{8}\left\|e_{v_{w q}}\right\|
\end{aligned}
$$

for all $e_{i_{g, d q}} \in \mathbb{B}_{e_{i_{g}, d q}}, e_{i_{w, d q}} \in \mathbb{B}_{e_{i_{w}, d q}}$ and $e_{v_{w, d q}} \in \mathbb{B}_{e_{v_{w}, d q}}$. Note that, in general, larger size of $\mathbb{B}_{(.)}$leads to larger values of $\beta_{k}$.

Applying the above inequalities and (45) to (44), we then get that:

$$
\begin{aligned}
\left\|\frac{d e_{z}}{d t}\right\| & \leq\|\Phi\| \cdot\left\|e_{z}\right\|+\alpha_{0}\left\|e_{z}\right\|+\beta_{1}\left\|e_{i_{g d}}\right\|+\beta_{2}\left\|e_{i_{g q}}\right\| \\
& +\beta_{3}\left\|e_{i_{w d}}\right\|+\beta_{4}\left\|e_{i_{w q}}\right\|+\beta_{5}\left\|e_{v_{w d}}\right\|+\beta_{6}\left\|e_{v_{w q}}\right\| \\
& +\beta_{7}\left\|e_{v_{w d}}\right\|+\beta_{8}\left\|e_{v_{w q}}\right\|
\end{aligned}
$$

holds for all $e_{z} \in \mathbb{B}_{e_{z}}, e_{i_{g, d q}} \in \mathbb{B}_{e_{i_{g, d q}}}, e_{i_{w, d q}} \in \mathbb{B}_{e_{i_{w, d q}}}$ and $e_{v_{w, d q}} \in \mathbb{B}_{e_{v_{w, d q}}}$.

Taking (34), (46) and (47) into account, it can be verified that there exist positive parameters $b_{k}^{i}$, for $k=1, \cdots, L+3$, $i \in \mathcal{N}$, and $c_{l}^{j}$, for $l=1, \cdots, 8, j \in \mathcal{M}$ such that $D_{2}$ satisfies the inequality:

$$
\begin{aligned}
& D_{2} \leq \sum_{i=1}^{N}\left[| e _ { i _ { g _ { i } d } } | \left(b_{1}^{i}\left|e_{i_{g_{i}}}\right|+b_{2}^{i}\left|e_{i_{g_{i} q}}\right|+b_{3}^{i}\left|e_{u_{c g_{i}}}\right|\right.\right. \\
& \left.\left.+\sum_{t=1}^{L} b_{t+3}^{i}\left|e_{i_{c_{t}}}\right|\right)\right]+\sum_{j=1}^{M}\left[\left(c_{1}^{j}\left|e_{i_{w_{j} d}}\right|+c_{2}^{j}\left|e_{i_{w_{j} q}}\right|\right)\right. \\
& \cdot\left(c_{3}^{j}\left|e_{v_{s w_{j} d}}\right|+c_{4}^{j}\left|e_{I v_{s w_{j} d}}\right|+c_{5}^{j}\left|e_{v_{s w_{j} q}}\right|+c_{6}^{j}\left|e_{I v_{s w_{j} q}}\right|\right. \\
& \left.\left.+c_{7}^{j}\left|e_{i_{w_{j} d}}\right|+c_{8}^{j}\left|e_{i_{w_{j} q}}\right|\right)\right]
\end{aligned}
$$

for all $e_{z} \in \mathcal{B}_{e_{z}} \subset \mathbb{B}_{e_{z}}, e_{i_{g, d q}} \in \mathcal{B}_{e_{i_{g}, d q}} \subset \mathbb{B}_{e_{i_{q}, d q}}, e_{i_{w, d q}} \in$ $\mathcal{B}_{e_{i_{w}, d q}} \subset \mathbb{B}_{e_{i_{w, d q}}}$ and $e_{v_{w, d q}} \in \mathcal{B}_{e_{v_{w, d q}}} \subset \mathbb{B}_{e_{v_{w, d q}}}$. These positive coefficients $\left(b_{k}^{i}\right.$ and $\left.c_{l}^{j}\right)$ are determined by the size of the domains $\mathcal{B}_{(\cdot)}$, the droop gains $k_{d_{i}}, k_{1,2 d_{j}}, k_{1,2 q_{j}}$, the system parameters and the prescribed setpoints while they are independent of $k_{1 g_{i}, d q}, k_{2 g_{i}, d q}, k_{1 w_{j}, d q}$ and $k_{2 w_{j}, d q}$.

Using Young's inequality :

$$
\|x\| \cdot\|y\| \leq \frac{1}{2 \mu}\|x\|^{2}+\frac{\mu}{2}\|y\|^{2}
$$

for the cross terms in (48) where $\mu$ is a positive constant that can be chosen arbitrarily, we obtain:

$$
D_{2} \leq \Gamma_{3}+\Gamma_{4}
$$

with

$$
\begin{aligned}
\Gamma_{3} & =\sum_{i=1}^{N}\left[\frac{b_{1}^{i}}{2} \nu_{1}^{i}\left|e_{i_{g_{i}} \mid}\right|^{2}+\frac{b_{2}^{i}}{2 \nu_{2}^{i}}\left|e_{i_{g_{i} q}}\right|^{2}\right] \\
& +\sum_{j=1}^{M}\left[\frac{d_{1}^{j}}{2} \kappa_{1}^{j}\left|e_{i_{w_{j}} d}\right|^{2}+\frac{d_{2}^{j}}{2} \kappa_{2}^{j}\left|e_{i_{w_{j} q}}\right|^{2}\right.
\end{aligned}
$$

and

$$
\begin{aligned}
\Gamma_{4} & =\sum_{i=1}^{N}\left[\frac{b_{3}^{i}}{2 \nu_{3}^{i}}\left|e_{u_{c g_{i}}}\right|^{2}+\sum_{t=1}^{L} \frac{b_{t+3}^{i}}{2 \nu_{t+3}^{i}}\left|e_{i_{c}}\right|^{2}\right] \\
& +\sum_{j=1}^{M}\left[\frac{d_{3}^{j}}{2 \kappa_{3}^{j}}\left|e_{v_{s w_{j} d}}\right|^{2}+\frac{d_{4}^{j}}{2 \kappa_{4}^{j}}\left|e_{I v_{s w_{j} d}}\right|^{2}\right. \\
& \left.+\frac{d_{5}^{j}}{2 \kappa_{5}^{j}}\left|e_{v_{s w_{j} q}}\right|^{2}+\frac{d_{6}^{j}}{2 \kappa_{6}^{j}}\left|e_{I v_{s w_{j} q}}\right|^{2}\right]
\end{aligned}
$$

where $\nu_{(\cdot)}$ and $\kappa_{(\cdot)}$ have the same role as $\mu$, that can be chosen arbitrarily.

In order to make the derivative of $W$ negative except at the origin, we first determine the values of droop gains $k_{d_{i}}, k_{1,2 d_{j}}$, $k_{1,2 q_{j}}$ and the size of region of attraction

$$
\mathcal{R}_{a t t}=\mathcal{B}_{e_{i_{g}, d q}} \times \mathcal{B}_{e_{i_{w}, d q}} \times \mathcal{B}_{e_{v_{w}, d q}} \times \mathcal{B}_{e_{z}}
$$

Then, the values of $b_{k}^{i}$ and $c_{l}^{j}$ can be estimated. Subsequently, we choose large $\nu_{(\cdot)}$ and $\kappa_{(\cdot)}$ such that $\Gamma_{1}+\Gamma_{4}<0$. Once $k_{d_{i}}, k_{1,2 d_{j}}, k_{1,2 q_{j}}$ are set, the size of region of attraction $\mathcal{R}_{a t t}$, $\nu_{(\cdot)}$ and $\kappa_{(\cdot)}$ are determined, no matter what values of $\frac{b_{1}^{i}}{2} \nu_{1}^{i}$, 
$\frac{b_{2}^{i}}{2} \nu_{2}^{i}, \frac{d_{1}^{j}}{2} \kappa_{1}^{j}$ and $\frac{d_{2}^{j}}{2} \kappa_{d}^{j}$ are, we can always find $\frac{b_{1}^{i}}{2} \nu_{1}^{i}<k_{1 g_{i} d}$, $\frac{b_{2}^{i}}{2} \nu_{2}^{i}<k_{1 g_{i} q}, \frac{d_{1}^{j}}{2} \kappa_{1}^{j}<k_{1 w_{j} d}$ and $\frac{d_{2}^{j}}{2} \kappa_{d}^{j}<k_{1 w_{j} q}$ and hence, $\Gamma_{2}+\Gamma_{3}<0$. Consequently, there exist $k_{1 g_{i} d}, k_{2 g_{i} q}, k_{1 w_{j} d}$, $k_{2 w_{j} q}, k_{1,2 d_{j}}$ and $k_{1,2 q_{j}}$ such that the derivative of $W$ is non-negative. In addition, $\dot{W}=0$ contains no trajectory of the system except the trivial trajectory. All solutions starting from $\mathcal{R}_{a t t}$ will converge to the origin. According to LaSalle theorem, the origin of the error system described by (32) is locally asymptotically stable. Thereby, the equilibrium $\bar{S}$ of the original closed-loop system (24) is also locally asymptotically stable. Finally, we can say that the control strategy can ensure the asymptotic stability of the MTDC system.

Remark 13: There is a trade-off between the size of $\mathcal{R}_{a t t}$ and the performance of system. If we want to get a large region of attraction, then we will have large values of $k_{1 g_{i}, d q}$ and $k_{1 w_{j}, d q}$. As seen in (12), large $k_{1 g_{i}, d q}$ and $k_{1 w_{j}, d q}$ maybe lead to large peak values of the control variables.

Remark 14: For the control algorithms described by (6), (16), (18) and (19), the control gains can be divided into two groups based on different control loops. It is clear that not all arbitrary positive control gains can ensure the system's stability. According to the lemmas and theorems derived from this section, the design guidelines for the control gains can be summarized as follows to guarantee system's stability.

- The control gains of fast control loop can be chosen as $k_{1,2 g_{i} d, q}, k_{1,2 w_{j} d, q} \in \mathbb{B}_{g_{f}}=\left\{k \in \mathbb{R} \mid k<\frac{1}{\epsilon_{f}}\right\}$ where $\epsilon_{f}$ is a small positive constant.

- The control gains of slow control loop can be chosen as $k_{1,2 d_{j}}, k_{1,2 q_{j}} \in \mathbb{B}_{g_{s}}=\left\{k \in \mathbb{R} \mid k<\frac{1}{\epsilon_{s}}\right\}$ where $\epsilon_{s}$ is a small positive constant.

- $\epsilon_{f}$ and $\epsilon_{s}$ are chosen such that $\epsilon_{f}<<\epsilon_{s}$.

- The droop gains are chosen such that (37) and (38) can be satisfied.

\section{Simulation STUdies}

In this section, the MTDC system as depicted in Fig. 3 consisting of two WVSCs and two GVSCs is simulated. The values of the parameters are listed in Table I and Table II. The AC voltage amplitude of the GVSCs is $415 \mathrm{~V}$. The base quantities of the per-unit system applied to the simulations are presented in Table III. The base quantities of the $\mathrm{AC}$ and DC currents are calculated as $I_{\text {ac,base }}=S_{\text {ac,base }} /\left(\sqrt{3} V_{\text {ac,base }}\right)$ and $I_{\mathrm{dc} \text {,base }}=S_{\mathrm{dc} \text {,base }} / V_{\mathrm{dc} \text {,base. As described in the previous }}$ section, the wind farm is modeled as a controlled current source and hence, $I_{w_{1}, d q}$ and $I_{w_{2}, d q}$ represent the power productions of the wind farms. The setpoints and the initial values of $I_{w_{1,2} d}$ are given by Table IV. $I_{w_{1} q}$ and $I_{w_{2} q}$ are set to zero. Furthermore, some system variables' initial values are provided by Table $\mathrm{V}$.

For all the simulations in this section, the integral parts of the fast control gains are set to zero, i.e. $k_{2 g_{i}, d q}=k_{2 w_{j}, d q}=0$. Three different sets of control gains as presented in Table VI are chosen to verify the theoretical analysis.

\section{A. Verification of two-time-scale behavior}

The control gains in Set 1 are considered for the converters in this part. The simulation results are displayed in Fig. 4.
TABLE I

PARAMETERS OF THE DC NETWORK.

\begin{tabular}{|c||c|c|}
\hline & Resistance $R_{c}$ & Inductance $L_{c}$ \\
\hline \hline$l_{1}$ & $0.01 \Omega$ & $6 \mathrm{mH}$ \\
\hline$l_{2}$ & $0.02 \Omega$ & $12 \mathrm{mH}$ \\
\hline$l_{3}$ & $0.15 \Omega$ & $9 \mathrm{mH}$ \\
\hline$l_{4}$ & $0.14 \Omega$ & $8.4 \mathrm{mH}$ \\
\hline$l_{5}$ & $0.16 \Omega$ & $9.6 \mathrm{mH}$ \\
\hline$l_{6}$ & $0.18 \Omega$ & $10.8 \mathrm{mH}$ \\
\hline$l_{7}$ & $0.19 \Omega$ & $11.4 \mathrm{mH}$ \\
\hline
\end{tabular}

TABLE II

PARAMETERS OF THE VSC TERMINAL.

\begin{tabular}{|c||c|c|c|}
\hline & $R_{g}\left(R_{w}\right)$ & $L_{g}\left(L_{w}\right)$ & $C_{g}\left(C_{w}\right)$ \\
\hline \hline SAC 1 & $9.9 \mathrm{~m} \Omega$ & $6 \mathrm{mH}$ & $68 \mu \mathrm{F}$ \\
\hline SAC 2 & $9.4 \mathrm{~m} \Omega$ & $12 \mathrm{mH}$ & $20 \mu \mathrm{F}$ \\
\hline WAC 1 & $8.4 \mathrm{~m} \Omega$ & $9 \mathrm{mH}$ & $27 \mu \mathrm{F}$ \\
\hline WAC 2 & $8.9 \mathrm{~m} \Omega$ & $8.4 \mathrm{mH}$ & $20 \mu \mathrm{F}$ \\
\hline
\end{tabular}

The trajectory of $i_{g_{1} d}$ in Fig. 4(a) clearly performs a twotime-scale behavior. It starts with a fast transient during the initial interval as shown in Fig. 4(b). After the decay of this fast dynamic, $i_{g_{1} d}$ is on or close to its manifold $i_{g_{1} d}^{*}$ in all future time. Figure 4(c) illustrates the error between $i_{g_{1} d}$ and $i_{g_{1} d}^{*}$. At the initial instant, $\left|i_{g_{1} d}-i_{g_{1} d}^{*}\right|$ is nearly 0.29 p.u.. After $t=0.06 \mathrm{~s}$, the discrepancy between the two trajectories is less than 0.015 p.u.. It turns out that during the initial interval $[0,0.06] \mathrm{s}$, the trajectory of $i_{g_{1} d}$ approaches that of $i_{g_{1} d}^{*}$. It is seen that, the exponential decay of the fast transient during the initial interval corresponds to the solution of the boundary-layer model. However, such two-time-scale behavior is not significant in the DC voltage and the AC voltage, which are considered to have slow dynamics. As depicted in Figs. $4(\mathrm{e})-4(\mathrm{~g})$, there is no apparent fast transient that can be found between the trajectories of $u_{c g_{1}}$ and $u_{c g_{1}}^{r e}\left(v_{s w_{1} d}\right.$ and $\left.v_{s w_{1} d}^{r e}\right)$. It can be observed that $u_{c g_{1}}$ and $v_{s w_{1} d}$ are well approximated by the solution of the reduced model, i.e. $u_{c g_{1}}^{r e}$ and $v_{s w_{1} d}^{r e}$. Moreover, $v_{s w_{1} d}$ asymptotically converges to its setpoins $v_{s w_{1} d}^{o}$ together with $v_{s w_{1} d}^{r e}$.

\section{B. Evaluation of the system performance in case of distur-} bance

At $t=2 \mathrm{~s}, I_{w_{1} d}$ is changed to 0.6 p.u. because of the increase in the active power generated by the wind farms.

TABLE III

BASE QUANTITIES USED IN THE PER-UNIT SYSTEM.

\begin{tabular}{|c||c|c|}
\hline AC side & $S_{\text {ac,base }}=4.5 \mathrm{kVA}$ & $V_{\mathrm{ac}, \text { base }}=415 \sqrt{3 / 2} \mathrm{~V}$ \\
\hline DC side & $S_{\mathrm{dc}, \text { base }}=3 \mathrm{kVA}$ & $V_{\mathrm{dc}, \text { base }}=700 \mathrm{~V}$ \\
\hline
\end{tabular}

TABLE IV

SETPOINTS AND INITIAL VALUES OF THE CURRENT SOURCE.

\begin{tabular}{|c|c|c|c|}
\hline & $P_{g_{i}}^{o}$ & $Q_{g_{i}}^{o}$ & $u_{g_{1}}^{o}$ \\
\hline SA 1 & $-0.4 S_{\text {ac,base }}$ & 0 & $V_{\text {dc,base }}$ \\
\hline SAC 2 & $-0.5 S_{\text {ac,base }}$ & 0 & $V_{\text {dc,base }}$ \\
\hline \hline & $v_{s w_{j} d}^{0}$ & $v_{s w_{j} q}^{0}$ & $I_{w_{j}} d$ \\
\hline WAC 1 & $V_{\text {ac,base }}$ & 0 & $0.4 I_{\text {ac,base }}$ \\
\hline WAC 2 & $V_{\text {ac,base }}$ & 0 & $0.5 I_{\text {ac,base }}$ \\
\hline
\end{tabular}


TABLE V

INITIAL STATES OF THE MTDC SYSTEM.

\begin{tabular}{|c|c|c|c|}
\hline$i_{g_{1} d}$ & $i_{g_{2} d}$ & $i_{w_{1} d}$ & $i_{w_{2} d}$ \\
\hline-0.2 p.u. & -0.1 p.u. & 0.3 p.u. & 0.3 p.u. \\
\hline \hline$v_{s w_{1} d}$ & $v_{s w_{1} q}$ & $v_{s w_{2} d}$ & $v_{s w_{2} q}$ \\
\hline 0.7 p.u. & 0.01 p.u. & 0.7 p.u. & 0.01 p.u. \\
\hline \hline$u_{c g_{1}}$ & $u_{c g_{2}}$ & $u_{c w_{1}}$ & $u_{c w_{2}}$ \\
\hline 1.043 p.u. & 1.043 p.u. & 1.043 p.u. & 1.043 p.u. \\
\hline
\end{tabular}

TABLE VI

CONTROL GAINS APPLIED TO THE MTDC SYSTEM.

\begin{tabular}{|c||c|c|c|}
\hline & $k_{d_{1}}$ & $k_{d_{2}}$ & $k_{1 g_{i}, d q}\left(k_{1 w_{j}, d q}\right)$ \\
\hline \hline Set 1 & $10 \cdot k_{d_{1 \mathrm{~min}}}$ & $10 \cdot k_{d_{2} \text { min }}$ & 1000 \\
\hline Set 2 & $10 \cdot k_{d_{1 \mathrm{~min}}}$ & $10 \cdot k_{d_{2 \mathrm{~min}}}$ & 300 \\
\hline Set 3 & $1 \cdot k_{d_{1 \mathrm{~min}}}$ & $2 \cdot k_{d_{2} \text { min }}$ & 1000 \\
\hline
\end{tabular}

Consequently, GVSC 1 and GVSC 2 should share the duty of eliminating the power unbalance caused by this increase of the power production. The simulation results are presented in Fig. 5. Since more power needs to be transmitted via the DC grid, in order to make the MTDC system operate normally, GVSC 1 and GVSC 2 should absorb more power from the DC grid. As shown in Figs. 5(a) and 5(b), $i_{g_{1} d}$ attains a new steady value around -0.5 p.u. from -0.4 p.u. and $\bar{i}_{g_{2} d}$ is also changed from -0.5 p.u. to -0.6 p.u.. As discussed in Remark 8 , if more power is absorbed by the GVSCs, this forces the DC voltages to rise and reach new steady levels. This phenomenon is clearly presented in Figs. 5(e) - 5(g). The new DC voltage transmission level now is about 1.05 p.u. which deviates from the setpoints $u_{c g_{1}, 2}^{o}=1.0$ p.u.. As seen in Fig. 5(c), $i_{w_{1} d}$ arrives around 0.6 p.u. to response to the change of $I_{w_{1} d}$. On the other hand, $v_{s w_{1} d}$ is always well controlled around its setpoint $v_{s w_{1} d}^{o}=1.0$ p.u. irrespective of the variation in $I_{w_{1} d}$ after a short transient as depicted in Fig. 5(h).

By contrast to the increase in $I_{w_{1} d}$, at $t=4 \mathrm{~s}, I_{w_{2} d}$ drops from 0.5 p.u. to 0.3 p.u.. The simulation results are summarized in Fig. 6. Since the generated power from the wind farm 2 decreases, $i_{w_{2} d}$ starts to decrease and then converges to a new steady state about 0.3 p.u. as shown in Fig. 6(c). From Figs. 6(a) - 6(b), $i_{g_{1} d}$ is changed from -0.5 p.u. to -0.4 p.u. and $i_{g_{2} d}$ varies from -0.6 p.u. to -0.5 p.u. This means less power is received by GVSC 1 and GVSC 2 because less power is injected into the DC grid. Additionally, in order to comply with the droop law (17), the DC voltages also drop and then get to a new steady state $(\sim 1.0$ p.u. $)$ as displayed in Figs. 6(d) - 6(f).

To evaluate the capability in terms of $\mathrm{AC}$ voltage regulation, at $t=6 \mathrm{~s}$, a new setpoint $v_{s w_{1} d}^{o}=0.9$ p.u. is sent to WVSC 1. Now $v_{s w_{1} d}$ is required to be stabilized around this new reference point. The transient response of $v_{s w_{1} d}$ is illustrated in Fig. 7(a). It is found that $v_{s w_{1} d}$ and $v_{s w_{1} d}^{r e}$ quickly converge to the new setpoint with an acceptable undershoot. Since $I_{w_{1} d}$ is unchanged during the interval $[6,7] \mathrm{s}$ as depicted in Fig. $7(\mathrm{~b})$, the change of $v_{s w_{1} d}^{o}$ has no effect on the steady state of $i_{w_{1} d}$. According to (5), less power flows through WVSC 1 due to the decrease of the AC voltage at the PCC, which implies that the total transmitted power reduces. Therefore, GVSC 1
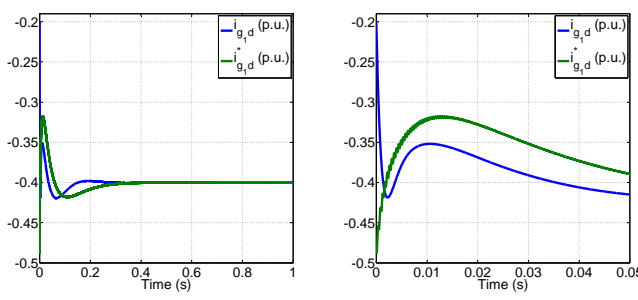

(a) Responses of $i_{g_{1} d}$ and (b) Zoom of $i_{g_{1} d}$ and $i_{g_{1} d}^{*}$. $i_{g_{1} d}^{*}$.
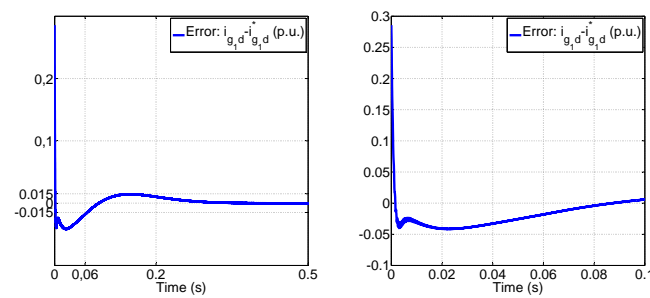

(c) Error between $i_{g_{1} d}$ and (d) Zoom of the error be$i_{g_{1} d}^{*}$. tween $i_{g_{1} d}$ and $i_{g_{1} d}^{*}$.
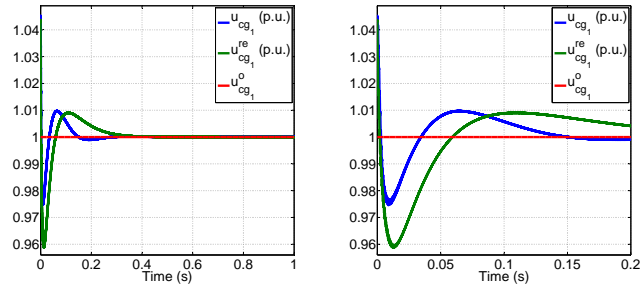

(e) Responses of $u_{c g_{1}}$ and (f) Zoom of $u_{c g_{1}}$ and $u_{c g_{1}}^{r e}$. $u_{c g_{1}}^{r e}$.
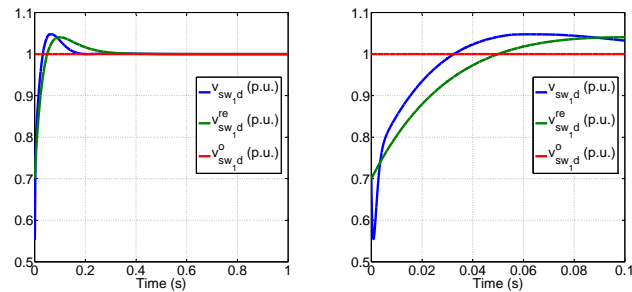

(g) Responses of $v_{s w_{1} d}$ and (h) Zoom of $v_{s w_{1} d}$ and $v_{s w_{1} d}^{r e}$. $v_{s w_{1} d}^{r e}$.

Fig. 4. Simulation results with the control gains in Set 1.

and GVSC 2 get less power than before and then, $i_{g_{1} d}$ and $i_{g_{2} d}$ start to decrease until converging to new steady states as seen in Figs. 7(c) and 7(d). Similar to the results in Figs. 6(d) - 6(f), the DC voltages start to drop and remain around 0.985 p.u. as shown in Figs. 7(e) and 7(f).

The responses of each terminal's active power at the PCC are plotted in Fig. 8. It is clear that GVSC 1 and GVSC 2 participate in balancing the active power of the DC grid. When the power injection grows, both GVSCs share the incremental power and then absorb more power from the grid. Conversely, as the power injection reduces, both GVSCs decrease their power absorption accordingly. 

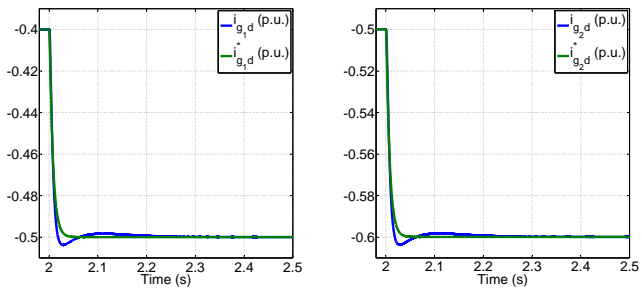

(a) Responses of $i_{g_{1} d}$ and (b) Responses of $i_{g_{2} d}$ and $i_{g_{1} d}^{*}$. $i_{g_{2} d}^{*}$.
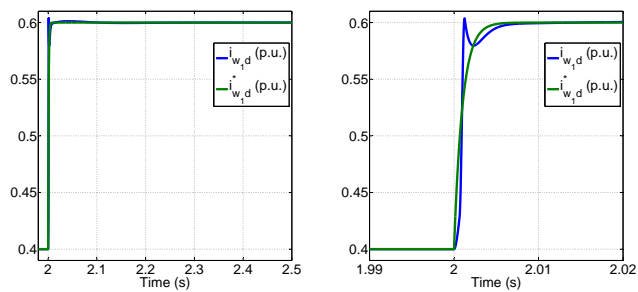

(c) Responses of $i_{w_{1} d}$ and (d) Zoom of $i_{w_{1} d}$ and $i_{w_{1} d}^{*}$. $i_{w_{1} d}^{*}$
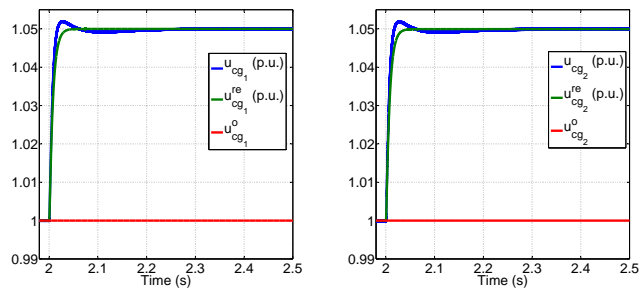

(e) Responses of $u_{c g_{1}}$ and (f) Responses of $u_{c g_{2}}$ and $u_{c g_{1}}^{r e}$.

$u_{c g_{2}}^{r e}$.
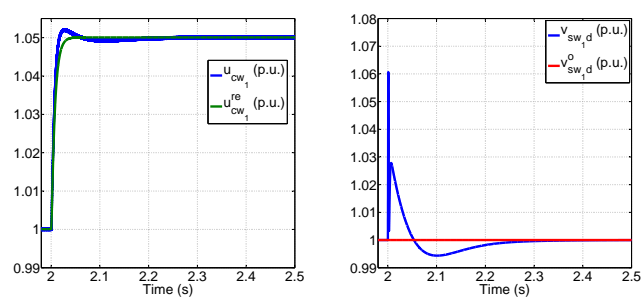

(g) Responses of $u_{c w_{1}}$ and $u_{c w_{1}}^{r e}$.

(h) Responses of $v_{s w_{1} d}$.

Fig. 5. Simulation results with the variation in $I_{w_{1} d}$.

\section{Dynamics regulation}

As stated in Remarks 10 and 12, we indicate that the control gains play different roles in regulating the system dynamics. This will be verified by the comparisons between different sets of the control gains.

We point out that the fast transient of the $d q$ currents corresponds to the solution of the boundary-layer model and then the fast dynamics can be regulated by the fast control gains. To clarify this issue, the comparison between Set 1 and Set 2 is carried out where $k_{1 g_{i}, d q}$ and $k_{1 w_{j}, d q}$ in Set 1 are larger than in Set 2. The simulation results are displayed in Fig. 9. During the initial interval, the two trajectories in Fig.
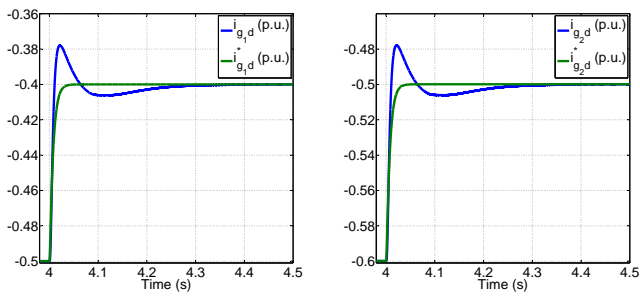

(a) Responses of $i_{g_{1} d}$ and (b) Responses of $i_{g_{2} d}$ and $i_{g_{1} d}^{*}$. $i_{g_{2} d}^{*}$.
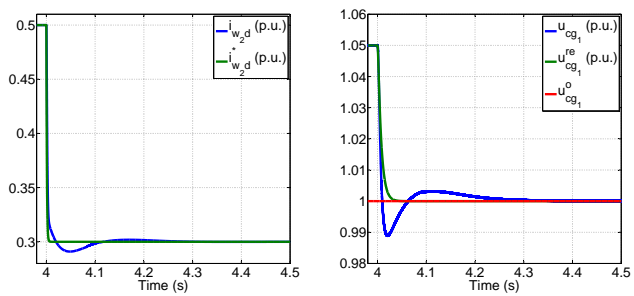

(c) Responses of $i_{w_{2} d}$ and (d) Responses of $u_{c g_{1}}$ and $i_{w_{2} d}^{*}$. $u_{c g_{1}}^{r e}$
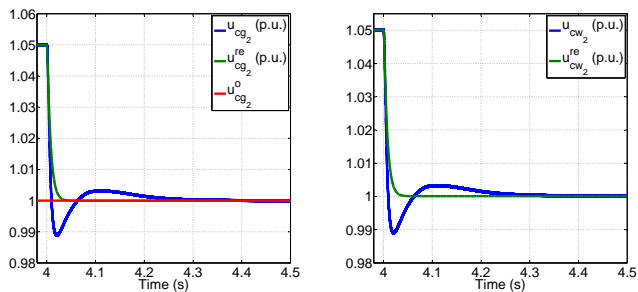

(e) Responses of $u_{c g_{2}}$ and (f) Responses of $u_{c w_{2}}$ and $u_{c g_{2}}^{r e}$. $u_{c w_{2}}^{r e}$.

Fig. 6. Simulation results with the variation in $I_{w_{2} d}$.

9(a) start from the same initial point around 0.285 p.u.. It is evident that the blue one has a faster rate of decay than that of the red one because of larger $k_{1 g_{1} d}$ in Set 1 than in Set 2. As seen in Fig .9(b), the blue curve enters the error band $[-0.005,0.005]$ p.u. around $t=0.25 \mathrm{~s}$ and then remains in this band in the future time, but in contrast, the red curve reaches this error band after $t=0.41 \mathrm{~s}$. A similar event occurs in the response of the error between $i_{w_{1} d}$ and $i_{w_{1} d}^{*}$ as plotted in Figs. 9(c) and 9(d). Both curves in Fig. 9(c) have the same initial value of 0.385 p.u.. Since $k_{1 w_{1} d}$ in Set 1 is larger than in Set 2, the response of the error represented by the blue curve drops faster than that described by the red one. Moreover, as seen in Fig. 9(d), after $t=0.2 \mathrm{~s}$, the blue curve stays in the error band $[-0.005,0.005]$ p.u.. The red one needs $0.41 \mathrm{~s}$ to get into this error band and then remains in it. The simulation results clearly show that the fast control gains play a critical role in the regulation of the fast transient of the $d q$ currents.

As expressed in (35), the behavior of the reduced model is regulated by the droop gains $k_{d_{i}}, k_{1, d q_{j}}$ and $k_{2, d q_{j}}$. Furthermore, the dynamics of $v_{s w_{j}, d q}$ in (35) heavily depend on $k_{1, d q_{j}}$ and $k_{2, d q_{j}}$, while the zero dynamics (or the dynamics of the DC network) strongly rely on the choice of the droop gains $k_{d_{i}}$. To demonstrate the above points, two sets of control 

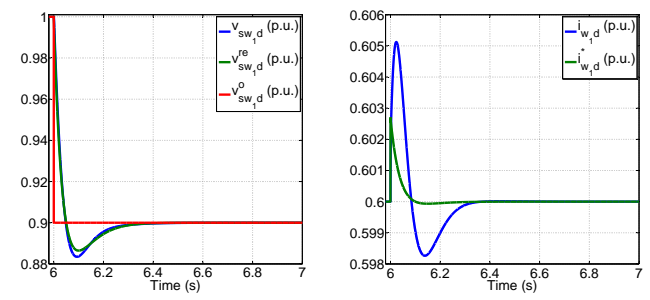

(a) Responses of $u_{s w_{1} d}$ and (b) Responses of $i_{w_{1} d}$ and $u_{s w_{1} d}^{r e}$. $\quad i_{w_{1} d}^{*}$.
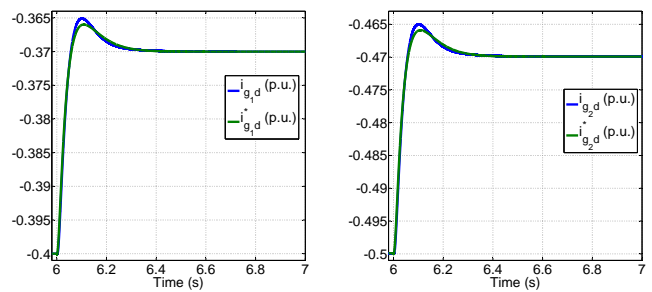

(c) Responses of $i_{g_{1} d}$ and (d) Responses of $i_{g_{2} d}$ and $i_{g_{1} d}^{*}$. $i_{g_{2} d}^{*}$.
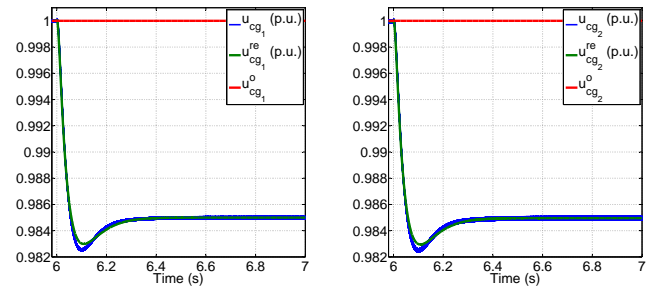

(e) Responses of $u_{c g_{1}}$ and (f) Responses of $u_{c g_{2}}$ and $u_{c g_{1}}^{r e}$.

$u_{c g_{2}}^{r e}$.

Fig. 7. Simulation results with AC voltage regulation.

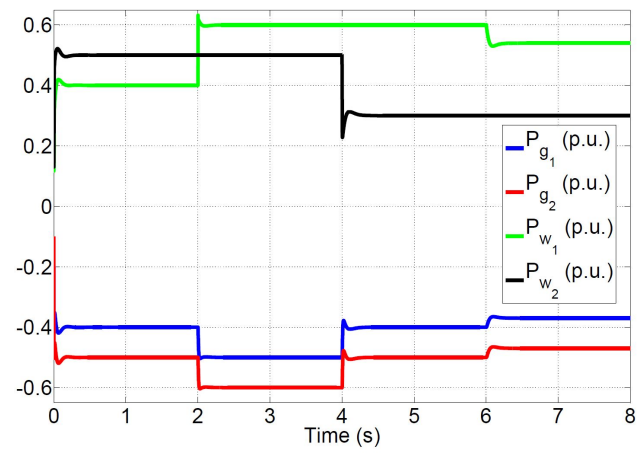

Fig. 8. Responses of active power during the interval $[0,8] \mathrm{s}$.

gains, i.e. Set 1 and Set 3, are chosen for comparison. The only difference between them is that $k_{d_{i}}$ in Set 1 is larger than in Set 3 .

The simulation results in Fig. 10 illustrate the effect of the droop gains on the system performance. From Figs. 10(a) and $10(\mathrm{c})$, both $u_{c g_{1}}$ and $u_{c w_{1}}$ with Set 1 and Set 3 are asymptotically stabilized around 1.0 p.u.. However, it can be observed that the blue trajectories remain in the domain $[0.99,1.01]$ p.u. after $t=0.05 \mathrm{~s}$, whereas the red ones need
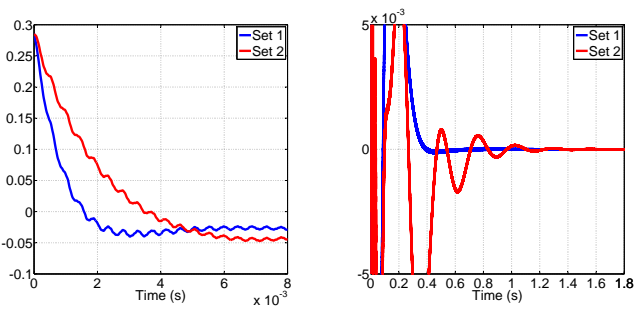

(a) Error between $i_{g_{1} d}$ and (b) Error between $i_{g_{1} d}$ and $i_{g_{1} d}^{*}$ during the initial inter- $i_{g_{1} d}^{*}$. val.
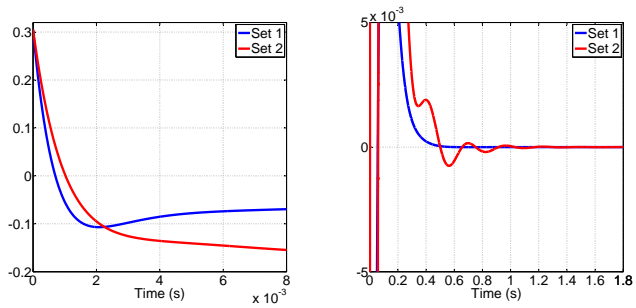

(c) Error between $i_{w_{1} d}$ and (d) Zoom of error between $i_{w_{1} d}^{*}$ during the initial inter- $i_{w_{1} d}$ and $i_{w_{1} d}^{*}$.
val.

Fig. 9. Comparison between Set 1 and Set 2.

nearly $1.9 \mathrm{~s}$ to stay in the same region. Moreover, as seen in Figs. 10(b) and 10(d), the response of $u_{c g_{1}}\left(u_{c w_{1}}\right)$ with Set 1 is much steeper than that with Set 2 during the initial interval. The above description indicates that the performance of the state variables related to the zero dynamics can be improved by increasing the values of $k_{d_{i}}$ appropriately.

Let us now focus on the responses of the $\mathrm{AC}$ voltage as depicted in Figs. 10(g) - 10(j). Interestingly, as seen in Fig. $10(\mathrm{~g})$, the response of $v_{s w_{1} d}$ with Set 1 is very close to that with Set 3. In particular, as seen in Fig. 10(h), the two curves, the blue one and the red one, almost coincide with each other. A similar result can also be obtained for $v_{s w_{2} d}$ from Figs. 10(i) and 10(j). This implies that the droop gains have little effect on controlling the $\mathrm{AC}$ voltage. It is $k_{1, d q_{j}}$ and $k_{2, d q_{j}}$ that are predominant in the regulation of the $\mathrm{AC}$ voltage.

\section{CONCLUSIONS}

This paper presents a detailed theoretical analysis of a control induced time-scale separation for an MTDC system with a generic DC grid topology using droop control strategy. The main contributions are that a rigorous mathematical explanation is provided for the dynamic separation in time scales between the system's state variables and notably, sufficient conditions on choosing the control gains for the system stability are derived using singular perturbation and Lyapunov theories. From the theoretical analysis, it is established that the time-scale separation can be created by the designed control algorithm. As a consequence, the system state variables can reasonably be partitioned into different dynamics where the $d q$ currents are listed as the fast dynamic state variables while the remaining variables have slow dynamics. Based on the time-scale separation, a boundary-layer and a reduced model 

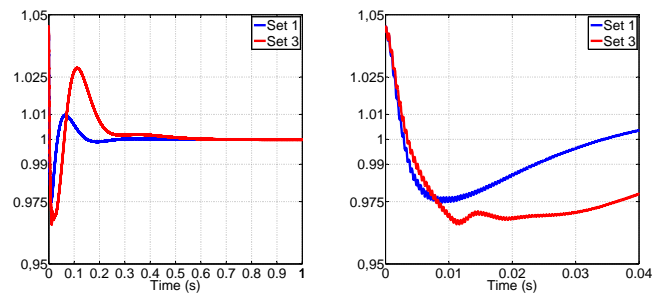

(a) Response of $u_{c g_{1}}$.

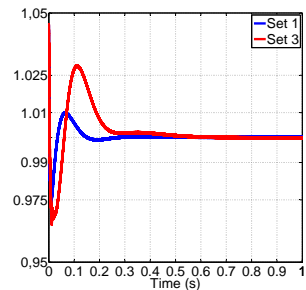

(c) Response of $u_{c w_{1}}$.

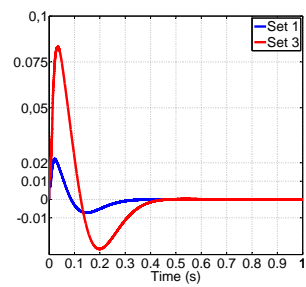

(e) Error between of $u^{r e}$ and $u_{c g_{1}}^{r e}$.
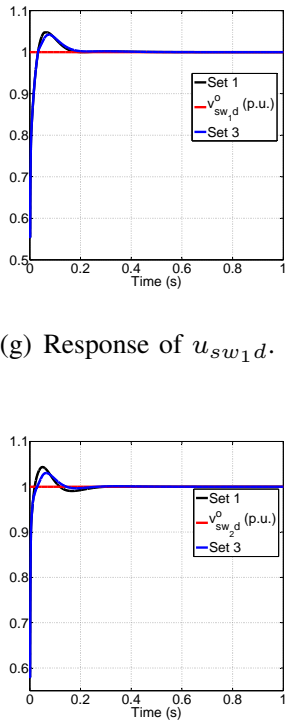

(i) Response of $u_{s w_{2} d}$.

(b) Response of $u_{c g_{1}}$ during the initial interval.

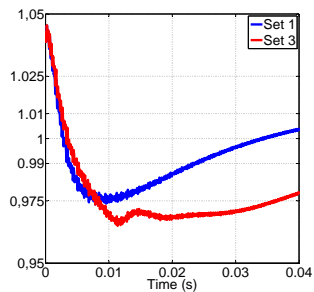

(d) Response of $u_{c w_{1}}$ during the initial interval.

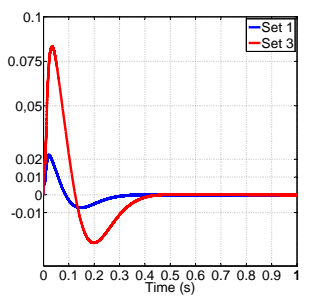

(f) Error between of $u_{c w_{1}}^{r e}$ and $u_{c w_{1}}^{r e}$.

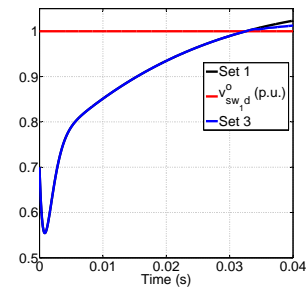

(h) Response of $u_{s w_{1} d}$ during the initial interval.

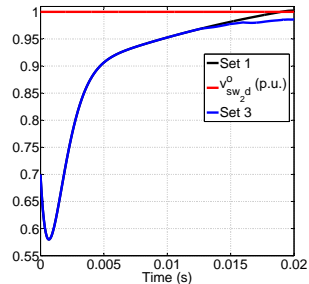

(j) Response of $u_{s w_{2} d}$ dur- (g) Response of $u_{s w_{1} d}$. ing the initial interval.

Fig. 10. Comparison between Set 1 and Set 3 .

are deduced. The exact solution of the slow dynamic state variables can be uniformly approximated by the solution of the reduced model. The fast transient of the fast dynamic state variables corresponds to the solution of the boundary-layer model. Additionally, our study establishes that the control gains in the fast control loop are mainly responsible for determining the fast transient of the $d q$ currents, while other gains are used to control the dynamics of the AC voltage. Finally, the droop gains have a great effect on the dynamics of the internal state variables (the dynamics of the DC grid).

The illustration of the theoretical analysis is carried out by numerical simulations. A sequence of events is applied to the MTDC system to evaluate the performance of the system with the proposed control structure. The simulation results clearly present that the $d q$ currents exhibit a significant two-time-scale behavior characterized by a slow and a fast transients. They also show that the control gains have different impacts on the behaviors of the system by performing the comparisons between three sets of control gains.

\section{REFERENCES}

[1] T. Haileselassie and K. Uhlen, "Power system security in a meshed north sea HVDC grid," Proceedings of the IEEE, vol. 101, no. 4, pp. 978-990, Apr. 2013.

[2] M. A. Abdelwahed and E. F. El-Saadany, "Power sharing control strategy of multiterminal vsc-hvdc transmission systems utilizing adaptive voltage droop," IEEE Transactions on Sustainable Energy, vol. 8, no. 2, pp. 605-615, Apr. 2017.

[3] A. Raza, X. Dianguo, S. Xunwen, L. Weixing, and B. W. Williams, "A novel multiterminal vsc-hvdc transmission topology for offshore wind farms," IEEE Transactions on Industry Applications, vol. 53, no. 2, pp. 1316-1325, Mar. 2017.

[4] S. Q. Bu, W. Du, H. F. Wang, Y. Liu, and X. Liu, "Investigation on economic and reliable operation of meshed mtdc/ac grid as impacted by offshore wind farms,' IEEE Transactions on Power Systems, vol. 32, no. 5, pp. 3901-3911, Sept 2017.

[5] S. Chondrogiannis and M. P. Blanco, "Market integration scheme of a multi-terminal hvdc grid in the north seas," IEEE Transactions on Power Systems, vol. 31, no. 3, pp. 2415-2422, May 2016.

[6] C. Stijn, "Steady-state and dynamic modelling of vsc hvdc systems for power system simulation," Ph.D. dissertation, Katholieke University Leuven, Belgium, 2010.

[7] T. M. Haileselassie, "Control, dynamics and operation of multi-terminal vsc-hvdc transmission systems," Ph.D. dissertation, Norwegian University of Science and Technology, 2012.

[8] Y. Chen, J. Dai, G. Damm, and F. Lamnabhi-Lagarrigue, "Nonlinear control design for a multi-terminal VSC-HVDC system," in 2013 European Control Conference (ECC), Jul. 2013, pp. 3536-3541.

[9] W. Wang and M. Barnes, "Power flow algorithms for multi-terminal VSC-HVDC with droop control," IEEE Transactions on Power Systems, vol. 29, no. 4, pp. 1721-1730, Jul 2014.

[10] A. Abdel-Khalik, A. Massoud, A. Elserougi, and S. Ahmed, "Optimum power transmission-based droop control design for multi-terminal HVDC of offshore wind farms," IEEE Transactions on Power Systems, vol. 28, no. 3, pp. 3401-3409, Aug. 2013.

[11] E. Prieto-Araujo, A. Egea-Alvarez, S. Fekriasl, and O. Gomis-Bellmunt, "Dc voltage droop control design for multiterminal hvdc systems considering ac and dc grid dynamics," IEEE Transactions on Power Delivery, vol. 31, no. 2, pp. 575-585, Apr 2016.

[12] F. Thams, R. Eriksson, and M. Molinas, "Interaction of droop control structures and its inherent effect on the power transfer limits in multiterminal vsc-hvdc," IEEE Transactions on Power Delivery, vol. 32, no. 1, pp. 182-192, Feb 2017.

[13] A. K. Marten, F. Sass, and D. Westermann, "Continuous p-vcharacteristic parameterization for multi-terminal hvdc systems," IEEE Transactions on Power Delivery, vol. 32, no. 4, pp. 1665-1673, Aug 2017.

[14] K. Rouzbehi, A. Miranian, J. I. Candela, A. Luna, and P. Rodriguez, "A generalized voltage droop strategy for control of multiterminal dc grids," IEEE Transactions on Industry Applications, vol. 51, no. 1, pp. 607-618, Jan 2015.

[15] N. Chaudhuri and B. Chaudhuri, "Adaptive droop control for effective power sharing in multi-terminal DC (MTDC) grids," IEEE Transactions on Power Systems, vol. 28, no. 1, pp. 21-29, Feb. 2013. 
[16] Y. Chen, G. Damm, A. Benchaib, and F. Lamnabhi-Lagarrigue, "Multitime-scale stability analysis and design conditions of a VSC terminal with DC voltage droop control for HVDC networks," in IEEE Conference on Decision and Control $(C D C)$. Los Angeles, CA, USA: IEEE, Dec. 2014.

[17] X. Chen, L. Wang, H. Sun, and Y. Chen, "Fuzzy logic based adaptive droop control in multiterminal hvdc for wind power integration," IEEE Transactions on Energy Conversion, vol. 32, no. 3, pp. 1200-1208, Sept 2017.

[18] G. Stamatiou and M. Bongiorno, "Power-dependent droop-based control strategy for multi-terminal hvdc transmission grids," IET Generation, Transmission Distribution, vol. 11, no. 2, pp. 383-391, 2017.

[19] E. Prieto-Araujo, F. Bianchi, A. Junyent-Ferre, and O. Gomis-Bellmunt, "Methodology for droop control dynamic analysis of multiterminal VSC-HVDC grids for offshore wind farms," IEEE Transactions on Power Delivery, vol. 26, no. 4, pp. 2476-2485, Oct 2011.

[20] P. Kokotovic, H. K. Khali, and J. O'reilly, Singular Perturbation Methods in Control: Analysis and Design. London, U.K.: Academic, 1986, vol. 25.

[21] G. Peponides, P. Kokotovic, and J. Chow, "Singular perturbations and time scales in nonlinear models of power systems," IEEE Transactions on Circuits and Systems, vol. 29, no. 11, pp. 758-767, Nov. 1982.

[22] J. Kimball and P. Krein, "Singular perturbation theory for DC-DC converters and application to PFC converters," IEEE Transactions on Power Electronics, vol. 23, no. 6, pp. 2970-2981, Nov. 2008

[23] S. Chandra, D. F. Gayme, and A. Chakrabortty, "Time-scale modeling of wind-integrated power systems," IEEE Transactions on Power Systems, vol. 31, no. 6, pp. 4712-4721, Nov 2016.

[24] E. C. Pillco and L. F. C. Alberto, "On the foundations of stability analysis of power systems in time scales," IEEE Transactions on Circuits and Systems I: Regular Papers, vol. 62, no. 5, pp. 1230-1239, May 2015.

[25] L. Xu, L. Yao, and C. Sasse, "Grid integration of large dfig-based wind farms using vsc transmission," IEEE Transactions on Power Systems, vol. 22, no. 3, pp. 976-984, Aug 2007.

[26] N. Mohan and T. M. Undeland, Power Electronics: Converters, Applications, and Design. John Wiley \& Sons Inc, 2003.

[27] L. Xu, B. Williams, and L. Yao, "Multi-terminal DC transmission systems for connecting large offshore wind farms," in IEEE Power and Energy Society General Meeting - Conversion and Delivery of Electrical Energy in the 21st Century, Jul 2008, pp. 1-7.

[28] K. Parthasarathy, Basic Graph Theory. Tata McGraw-Hill, 1994.

[29] J. A. Bondy and U. S. R. Murty, Graph theory with applications. Macmillan London, 1976, vol. 6.

[30] J. Beerten, S. Cole, and R. Belmans, "Modeling of multi-terminal VSC HVDC systems with distributed DC voltage control," IEEE Transactions on Power Systems, vol. 29, no. 1, pp. 34-42, Jan 2014.

[31] C. Du, A. Sannino, and M. Bollen, "Analysis of the control algorithms of voltage-source converter HVDC," in IEEE Power Tech, Jun. 2005, pp. 1-7.

[32] D.-C. Lee, G.-M. Lee, and K.-D. Lee, "DC-bus voltage control of threephase AC/DC PWM converters using feedback linearization," IEEE Transactions on Industry Applications, vol. 36, no. 3, pp. 826-833, May 2000.

[33] T. Haileselassie and K. Uhlen, "Impact of dc line voltage drops on power flow of MTDC using droop control," IEEE Transactions on Power Systems, vol. 27, no. 3, pp. 1441-1449, Aug. 2012.

[34] J. L. Thomas, S. Poullain, and A. Benchaib, "Analysis of a robust DC-bus voltage control system for a VSC transmission scheme," in Seventh International Conference on AC and DC Transmission, London, November 2001.

[35] M. J. Carrizosa, F. Navas, G. Damm, and F. Lamnabhi-Lagarrigue, "A two-time scale dynamic correction method for fifth-order generator model undergoing large disturbances," International Journal of Electrical Power \& Energy Systems, vol. 65, no. 5, pp. 291-298, Feb 2015.

[36] H. Khalil, Nonlinear Systems, 3rd ed. New Jersey: Prentice Hall, 1996.

[37] R. A. Horn and C. R. Johnson, Matrix Analysis. Cambridge university press, 1985.

[38] A. Isidori, Nonlinear Control Systems, Third Edition. Springer, 1995. 